\title{
PLIMPTON 322: \\ A UNIVERSAL CUNEIFORM TABLE FOR OLD BABYLONIAN MATHEMATICIANS, BUILDERS, SURVEYORS AND TEACHERS
}

\author{
Rudolf HaJOSSY
}

\begin{abstract}
This article deals with the damaged and incomplete Old Babylonian tablet Plimpton 322 which contains 4 columns and 15 rows of a cuneiform mathematical text. It has been shown that the presumed original table with its 7 columns and 39 rows represented: a table of square roots of numbers from 0 to 2 for mathematicians; an earliest rudiments of a trigonometric table for builders and surveyors where angles are not measured as an arc in a unit circle but as a side of a unit right-angled triangle; a list of the 39 exercises on reciprocal pairs, unit and integer-side right triangles (rectangles), factorization and square numbers for teachers.

The article provides new arguments in favor of old disputes (squares of diagonals or widths; mistakes in previous analysis of errors in P322). Contradictory ideas about P322 are discussed: Is it the table of triangle sides or factorization terms? Was it compiled by a parallel or independent factorization of the sides or of their squares? Are sides of an initial unit triangle enlarged or reduced by such a factorization? Does it contain two or four arithmetical errors?

Time and dimensional requirements for calculation and writing of the complete tablet have been also estimated.
\end{abstract}

\section{Introduction}

The cuneiform clay tablet Plimpton 322 (P322) [1, [8], [10, [11, Fig. 1, is very likely the most famous mathematical product of Old Babylonian era (1900-1600 $\mathrm{BCE}$ ), written probably in an ancient city Larsa (southern Iraq) several decades before the city was conquered (1762 BCE) by Hammurabi of Babylon. Opinions on the tablet have been gradually changing: After its illegal excavation, it was sold in 1923 for $\$ 10$ by a dealer E. J. B a n k to a collector G. A. P lim p t o n and in 1936 it was bequeathed to Columbia University, New York.

(c) 2016 Mathematical Institute, Slovak Academy of Sciences. 2010 Mathematics Subject Classification: 01A17.

Keyw ords: Old Babylonian cuneiform tablet, Plimpton 322, compilation, errors, users, sexagesimal numbers, Pythagorean theorem and triplets. 


\section{RUDOLF HAJOSSY}

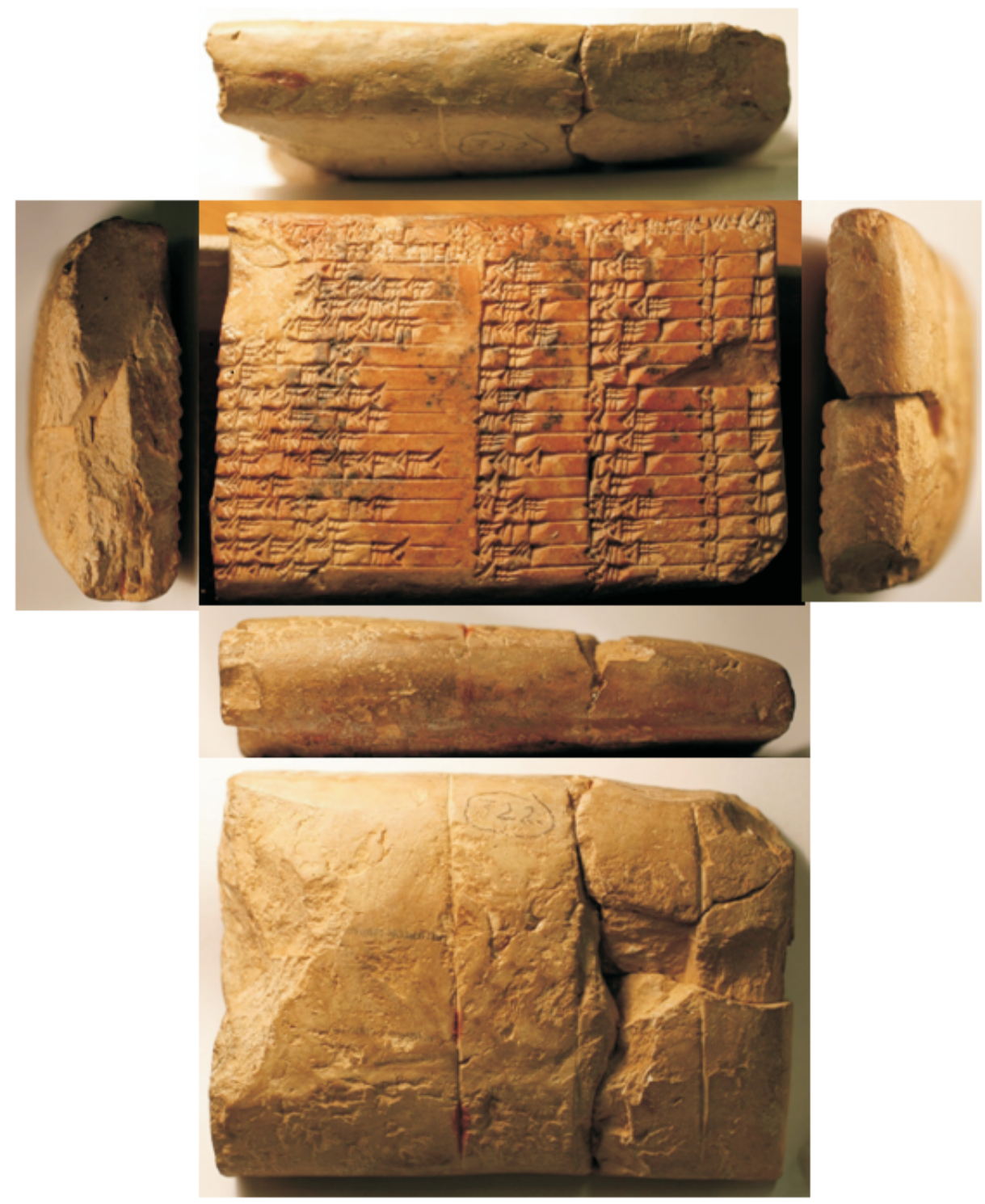

Figure 1. A. All sides of the cuneiform clay tablet Plimpton 322.

(The photo by Christine Proust from her online article [11 is published with her permission and by courtesy to Jane Spiegel, the librarian of the Rare Book and Manuscript Library, University of Columbia, New York.) 
PLIMPTON 322: A UNIVERSAL CUNEIFORM TABLE

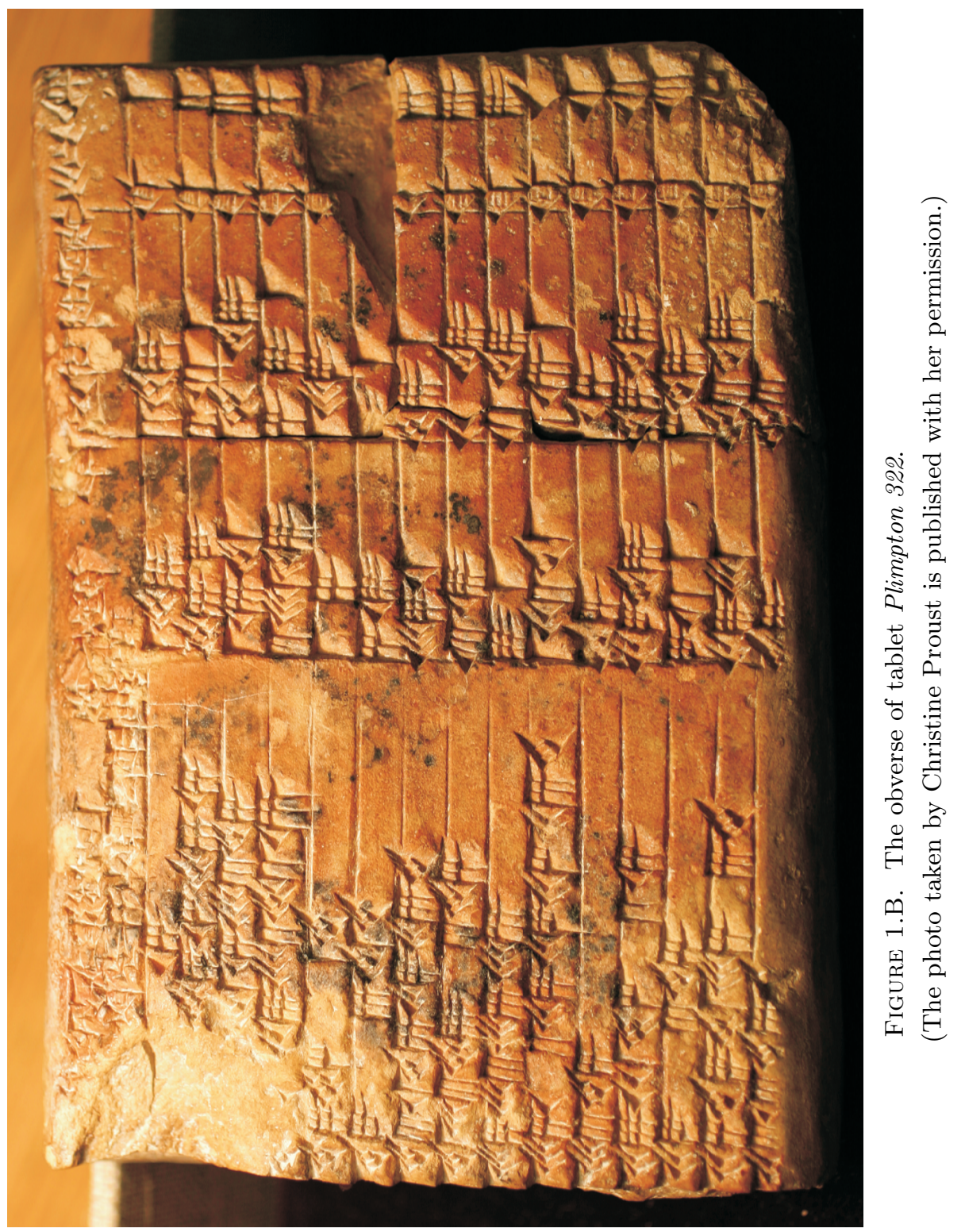


N e u g ebauer and Sachs (1945), 1] revealed mathematical importance of P322 and supposed that its text deals with "Pythagorean triangles (triplets)" obtained by generating pairs of regular numbers. B r u ins (1949), [2] showed that the triplets could be calculated also from reciprocal pairs and by reduction of common divisors. D e S oll a P r i c e (1964), 3] showed how to obtain necessary generating numbers or reciprocal pairs and assumed an original tablet with 38 rows of triangles. His conjecture was supported by the scored reverse side of the tablet (Fig. 1.A.), suitable to accommodate remaining rows of triangles. Friberg (1981), 4] reconstructed a complete extended table P322 combining the proposals of Bruins and de Solla Price. Høyrup (1990), 5] gave a geometrical interpretation of the Bruins algebraic relations between reciprocal pairs and right triangles, in accord with other cuneiform texts. J o y c e (1995), [6] interpreted P322 as a trigonometric table. R o b s o n (2001), [7] reconstructed the text of damaged headings in P322. She rejected the trigonometric interpretation and regarded the tablet, together with Friberg, as a teachers' aid. Casselman (2003), [8] briefly and clearly popularized P322, and showed how to read its cuneiform text in attached photo. B ritton, P r o us t and S hnider (2011), [10] offered a detailed review of a current knowledge on P322. Proust (2015), [11] published new high quality photos of all sides of P322 enabling to solve long lasting disputes.

The name Plimpton 322 denotes that it is the 322nd item in the catalogue of the university's cuneiform tablets where it is described [10] as a very large, well preserved, burned tablet (measuring some $13 \times 9 \mathrm{~cm}$; its thickness $2-3 \mathrm{~cm}$ ) with left-edge broken away, on obverse 4 columns and 16 lines, reverse blank; content: commercial account. Each of the four columns contains a heading in a mixture of Sumerian and Akkadian and 15 rows of numbers in a sexagesimal positional system with base 60 (Fig. 2).

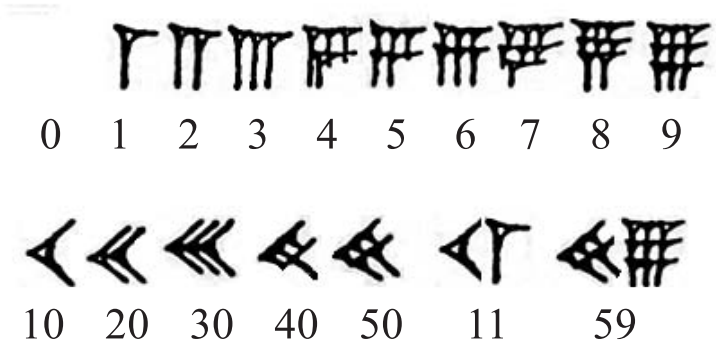

Figure 2. The cuneiform notation of sexagesimal digits: Zero is represented by a blank space. The numbers 1 up to 9 and also 10, 20, 30, 40, 50 have special characters. All other numbers are composed as a sum of these characters. For example, $11=10+1$ or $59=50+9$. 


\section{PLIMPTON 322: A UNIVERSAL CUNEIFORM TABLE}

Old Babylonians had no signs for zero or floating point - a boundary between the integer and fractional part of a sexagesimal number. The lack makes any interpretation of a written number to some extent an arbitrary one and leads easily to errors. For clarity, we write sexagesimal numerals from 0 to 59 as two-place decimal numerals and denote a floating point as a semicolon ";".

Multiplication of sexagesimal numbers is laborious as multiplication tables up to $100 ; \times 100$; are beyond the common memory. Division is even more challenging. This is the reason why the Old Babylonians instead of dividing by the number (igi) $x$ multiplied by its inverse (igibi) $1 / x$. They learned by heart The standard table of reciprocal pairs (Tab. 1).

TABLE 1 . The standard table of sexagesimal reciprocal pairs $x$ and $y=1 / x$ fulfilling the condition $x y=1$. Transcription of the cuneiform tablet $M L C 1670$ [12 in its original form: with the blank space instead of zero and the numbers in their relative form without a floating point. The absolute value of the numbers depends on the value of the number 1 . (Whether it is interpreted as $1 ;, 100 ; 0 ; 01$ or any other power of 60. )

\begin{tabular}{|c|c||c|c||c|l||r|c|}
\hline \multicolumn{2}{|c|}{$\begin{array}{c}\text { 2/3 of } 1 \text { is } 40 \\
\text { its } 1 / 2 \text { is } 30\end{array}$} & $\begin{array}{c}\boldsymbol{x} \\
\text { igi }\end{array}$ & $\begin{array}{c}\mathbf{1} / \boldsymbol{x} \\
\text { igibi }\end{array}$ & $\begin{array}{c}\boldsymbol{x} \\
\text { igi }\end{array}$ & $\begin{array}{c}\mathbf{1 / x} \\
\text { igibi }\end{array}$ & $\begin{array}{c}\boldsymbol{x} \\
\text { igi }\end{array}$ & $\begin{array}{c}\mathbf{1 / x} \\
\text { igibi }\end{array}$ \\
\hline $\boldsymbol{x}$ & $\mathbf{1} / \boldsymbol{x}$ & 9 & 640 & 24 & 230 & 45 & 120 \\
\hline igi & igibi & 10 & 6 & 25 & 224 & 48 & 115 \\
\hline 3 & 20 & 12 & 5 & 27 & 21320 & 50 & 112 \\
\hline 4 & 15 & 15 & 4 & 30 & 2 & 54 & 1240 \\
\hline 5 & 12 & 16 & 345 & 32 & 15230 & 1 & 1 \\
\hline 6 & 10 & 18 & 320 & 36 & 140 & 1 & 5615 \\
\hline 8 & 730 & 20 & 3 & 40 & 130 & 121 & 442640 \\
\hline
\end{tabular}

The table is readable in both directions: e.g., the pair $x=3$ and $1 / x=20$ corresponds to the pair $x=30$ and $1 / x=2$, and vice versa. It enables to extend the table easily by double-digit numbers, since, e.g., the pair $x=50,1 / x=112$ corresponds to the inverted pair $x=112,1 / x=50$.

The ancient mathematicians also knew relations between reciprocal pairs $x, y$ and sides $s, d, h$ of right triangles (rectangles) used by then builders and surveyors (Fig. 3 and 4). (Old Babylonians preferred rectangles with 2 sides and diagonal, but for simplicity, we will talk about triangles with 3 sides.)

As it follows from the Fig. 3 and 4 , the diagonal $d$ (siliptum in Akkadian), the width $s$ (sag in Sumerian) and the length $h$ (uš in Sumerian) of a rectangle 

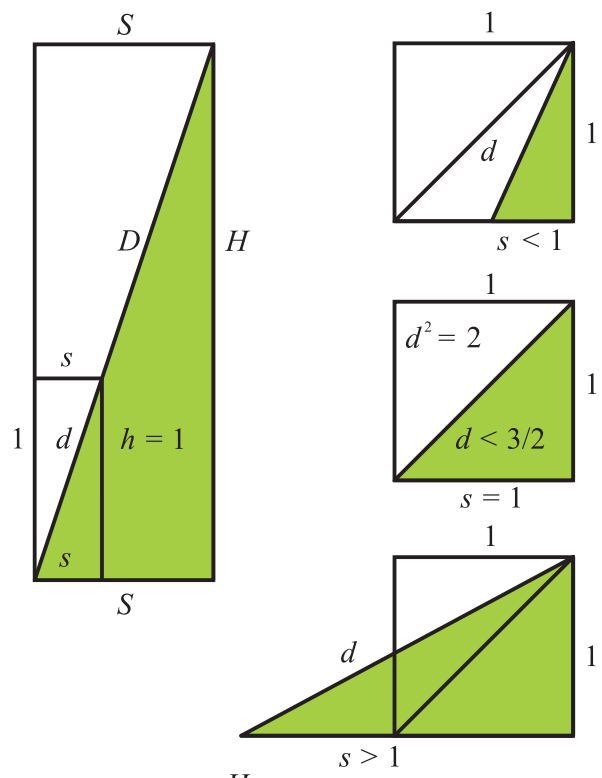

$H$

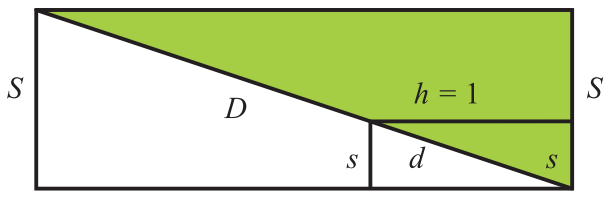

$H$

FiguRE 3. Right-angled triangles used by Old Babylonian builders or surveyors:

On the left: A field, a steep wall of a building or a channel with a triangular cross-section $(S, D, H)$. A similar unit (measuring) triangle with sides $s, d, h=1$. Each side had a Sumerian-Akkadian naming: $s a g=$ width $(S, s)$, şiliptum $=$ diagonal $(D, d), u \check{s}=$ length $(H, h)$.

On the right: Unit triangles with different slopes $s$ of diagonals $d$ : a steep slope $(s<1)$, a gentle slope $(s>1)$. The diagonal of unit square has the slope $s=1$. (The slope $=$ śágal in Sumerian.)

In the bottom: A simple turn of a steep triangle changes it to one with a gentle slope as in a case of embankments or staircases.

or a right triangle obey the following relations

$$
\begin{aligned}
& d^{2}-s^{2}=h^{2}, \\
& d^{2}-s^{2}=x y=(d+s)(d-s) .
\end{aligned}
$$

The "diagonal" rule (11) enables to calculate one side of the right-angled triangle. (1000 years before the birth of Pythagoras, it would seem ridiculous to call the rule "Pythagorean".) 
PLIMPTON 322: A UNIVERSAL CUNEIFORM TABLE
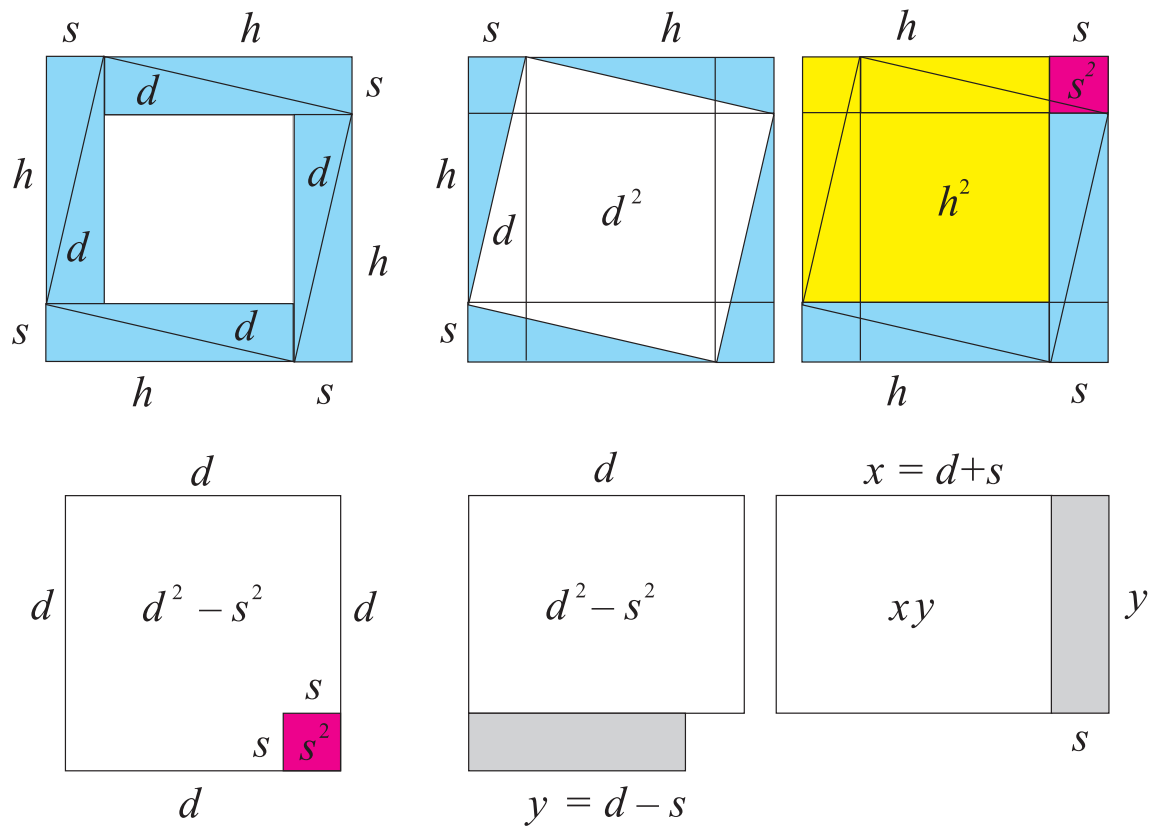

Figure 4. Geometric relations between the sides $s, h, d$ of a right-angled (blue) triangle obtained by means of the square $(s+h)^{2}$ with the sides $s+h$ (top picture) or by the difference of squares $d^{2}-s^{2}$ (bottom picture). The specific relations can be briefly, though anachronistically, expressed by modern algebraic relations:

Upper pictures: $(s+h)^{2}=d^{2}+4(1 / 2 s h)=h^{2}+s^{2}+4(1 / 2 s h)$ and therefore $d^{2}=h^{2}+s^{2}, s^{2}=d^{2}-h^{2}, d^{2}-s^{2}=h^{2}$.

Lower pictures: $d^{2}-s^{2}=x y=(d+s)(d-s)$, while $x=d+s, y=d-s$ or $d=(x+y) / 2, s=(x-y) / 2$.

According to the relations (11) and (2), in case of a unit right triangle with the length $h=1$, the sides

$$
x=d+s, \quad y=d-s
$$

of a rectangle with the unit area

$$
x y=h^{2}=1^{2}
$$

represent a reciprocal pair $x, y=1 / x$ determined by the diagonal $d$ and the width $s$ of the unit triangle.

Inversely, as it follows from (3), the sides

$$
d=(x+y) / 2, \quad s=(x-y) / 2, \quad h=1
$$

of a unit triangle could be determined by a reciprocal pair $x, y$. 
The set of relations (4), (5) can be written in a form of modern quadratic equations

$$
x^{2}-2 d x+1=0 \quad \text { or } \quad x^{2}-2 s x-1=0
$$

for an unknown number $x$ and known values of the diagonal $d$ or the width $s$. Of course, Old Babylonians solved the quadratic equations (6) for an unknown reciprocal pair $x, y$ in a different way as we would do:

At first, knowing the diagonal $d$, they calculated its square $d^{2}$. Gradually, using the "diagonal" rule (1), the square of width $s^{2}=d^{2}-1^{2}$ and then its square root, i.e., the unknown width $s=\sqrt{d^{2}-1}$, were calculated. Eventually, using relations (3) for already known pair of sides $d, s$, they calculated the searched reciprocal pair

$$
x=d+s=d+\sqrt{d^{2}-1}, \quad y=d-s=d-\sqrt{d^{2}-1} .
$$

In another type of equation (6), with the known width $s$, by a similar procedure and relations (11) and (3), they obtained the reciprocal pair $x=\sqrt{s^{2}+1}+s, y=\sqrt{s^{2}+1}-s$.

To obtain these solutions, it was necessary to calculate squares $d^{2}$ or $s^{2}$ and then values of the square roots $s=\sqrt{d^{2}-1^{2}}$ or $d=\sqrt{1^{2}+s^{2}}$ (probably, using a suitable table). Since in this case the roots represented sides $s, d$ of a unit triangle then such a table of square roots could be obtained by the relations (5) from a set of reasonable chosen pairs of reciprocals $x, y$.

It was natural to use at first the simple, well-known reciprocal pairs $x, y$ from The standard table (Tab. 1) for calculation of sides $s=(x-y) / 2, d=(x+y) / 2$, $h=1$ of unit triangles and their squares $s^{2}$ or $d^{2}=1+s^{2}$. The results of such calculations for the values $x$ from 1 ; to 3 ; are shown in Tab. 2. This preliminary table does not represent any known cuneiform tablet. In spite of this, all its data, except of those yellow colored, can be found in the Old Babylonian tablets $M S$ 3502 (the 8th row of Tab. 2), MS 3971 (the 2nd to 7th row) and partly in the P322 (the 8th and 9th row) (Friberg (2007), 9]). The green numbers or letters, shown in Tab. 2, substitute the missing original numbers or letters in damaged parts of the corresponding clay tablets. Headings in Tab. 2 (in Sumerian-Akkadian mixture) for the parameters $x, y, s, d$, use the same words as tablets $M S$ 3502, $M S$ 3971. The rest of headings is in agreement with P322 and is explained in Fig. 5. The squares of the width $s^{2}$ or the diagonal $d^{2}=1+s^{2}$ in Tab. 2 could have been calculated in several ways:

- By the generating reciprocal pair $x, y$ as

$$
\begin{aligned}
& s^{2}=[(x-y) / 2]^{2}=\left(x^{2}+y^{2}\right) / 4-1 / 2, \\
& d^{2}=[(x+y) / 2]^{2}=\left(x^{2}+y^{2}\right) / 4+1 / 2 .
\end{aligned}
$$


PLIMPTON 322: A UNIVERSAL CUNEIFORM TABLE

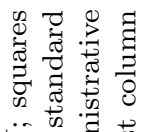

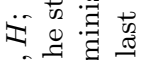

入北

के द्व

y

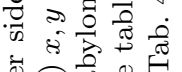

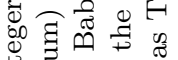

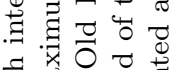
돌 की वै $\frac{0}{60}+\frac{1}{4}: 0$

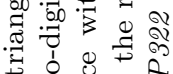

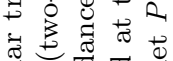

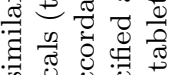
की

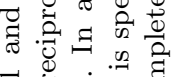
- $-\dot{0}$ के 110 每 $\approx=$ \% क क्ष

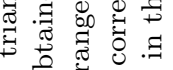

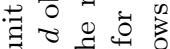

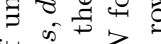
茯

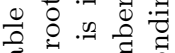
क ष \&

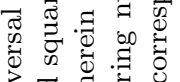

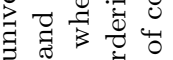
o $\approx \hat{-1}$ क ज त $\frac{1}{4}$ 兘

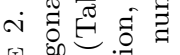
国.

\begin{tabular}{|c|c|c|c|c|c|c|c|c|c|c|c|c|}
\hline $\begin{array}{l}\text { खै } \\
\text { है }\end{array}$ & $z$ & ल) & 的 & กิ & $\ddot{\sigma}$ & จ & $\stackrel{N}{N}$ & $\stackrel{\infty}{-1}$ & 그 & $\neg$ & & \\
\hline हే हే & $z_{1}$ & - & N & $\infty$ & 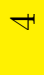 & مL & 0 & $\sim$ & $\infty$ & $a$ & $\stackrel{ }{\circ}$ & 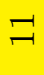 \\
\hline 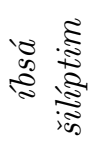 & $A \underset{\gamma}{\mathbb{Z}}$ & -1 & $\begin{array}{l}\sigma \\
\infty\end{array}$ & $\begin{array}{l}5 \\
-1\end{array}$ & $\not$ & $\stackrel{10}{\sim}$ & $\stackrel{\infty}{-1}$ & क & 10 & $\begin{array}{l}\underset{+}{+} \\
\text { N }\end{array}$ & $\stackrel{\sim}{\sim}$ & D \\
\hline $\begin{array}{ll}i & \mathbb{2} \\
\infty & 8 \\
0 & 0\end{array}$ & is $\underset{\infty}{N}$ & 0 & $\vec{n}$ & 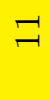 & $\sigma$ & 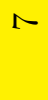 & 10 & $\stackrel{0}{0}$ & $\infty$ & $\begin{array}{l}8 \\
10 \\
-1\end{array}$ & $\vec{N}$ & $\nabla^{\prime}$ \\
\hline 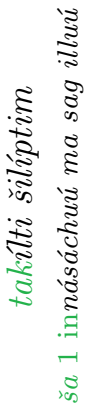 & 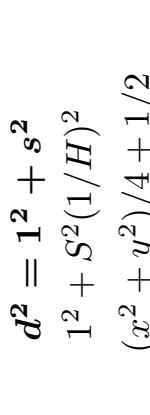 & $\stackrel{8}{\stackrel{8}{-1}}$ & $\begin{array}{l}10 \\
-1 \\
0 \\
10 \\
8 \\
0 \\
10 \\
-1 \\
8 \\
0 \\
-1\end{array}$ & 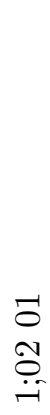 & 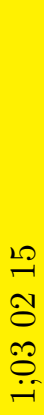 & $\begin{array}{l}\stackrel{10}{1} \\
0 \\
0 \\
20 \\
\stackrel{0}{-1}\end{array}$ & $\begin{array}{l}\stackrel{20}{2} \\
\stackrel{0}{\cdots} \\
\stackrel{-}{-1}\end{array}$ & $\begin{array}{l}\stackrel{\Xi}{0} \\
\stackrel{N}{\ddot{H}}\end{array}$ & 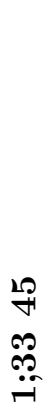 & 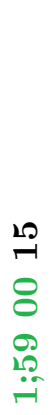 & $\begin{array}{l}9 \\
0 \\
0 \\
\stackrel{0}{1}\end{array}$ & $\begin{array}{l}\stackrel{0}{4} \\
0 \\
\stackrel{+}{+1} \\
\dot{v}\end{array}$ \\
\hline 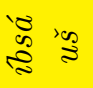 & $\mathbb{I}$ & - & $\begin{array}{l}8 \\
\infty\end{array}$ & 8 & 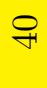 & $\stackrel{H}{N}$ & $\stackrel{\sim}{\sim}$ & ? & $\forall$ & $\begin{array}{l}8 \\
\text { N }\end{array}$ & $\stackrel{\overbrace{}}{\sim}$ & $\infty$ \\
\hline 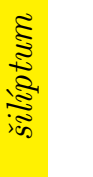 & 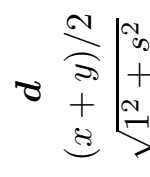 & $\stackrel{8}{\stackrel{8}{-}}$ & 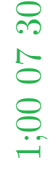 & $\stackrel{-1}{\circ}$ & 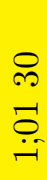 & 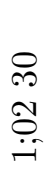 & $\stackrel{\mathscr{2}}{\stackrel{2}{-1}}$ & $\stackrel{\infty}{\stackrel{0}{0}}$ & $\stackrel{10}{\stackrel{20}{-1}}$ & $\begin{array}{l}\stackrel{\odot}{\infty} \\
\stackrel{\leftrightarrow}{+} \\
\stackrel{\sim}{\leftarrow}\end{array}$ & $\stackrel{N}{\stackrel{N}{-1}}$ & $\stackrel{0}{\stackrel{+}{-1}}$ \\
\hline 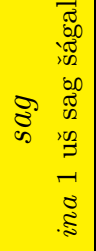 & 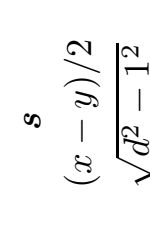 & $\ddot{0}$ & 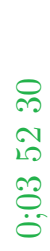 & $\ddot{F}$ & $\begin{array}{l}\stackrel{0}{m} \\
\ddot{n} \\
\ddot{0}\end{array}$ & $\begin{array}{l}\stackrel{\circ}{\infty} \\
\stackrel{1}{0}\end{array}$ & 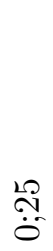 & $\stackrel{\overbrace{}}{0}$ & $\stackrel{12}{4}$ & $\begin{array}{l}\stackrel{8}{\infty} \\
0 \\
\stackrel{0}{0}\end{array}$ & $\stackrel{\leftrightarrow}{\stackrel{3}{-1}}$ & $\stackrel{尺}{\stackrel{\overbrace{}}{\sim}}$ \\
\hline . & 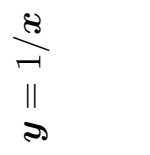 & $\ddot{-}$ & $\begin{array}{l}10 \\
10 \\
0 \\
0 \\
0\end{array}$ & $\stackrel{0}{\circ}$ & $\stackrel{\infty}{\stackrel{\infty}{+}}$ & $\stackrel{10}{+1}$ & $\stackrel{\circ}{\stackrel{+}{0}}$ & $\ddot{0}$ & $\stackrel{8}{\circ}$ & $\stackrel{\mathscr{L}}{\stackrel{0}{0}}$ & $\stackrel{\vec{N}}{0}$ & $\stackrel{尺}{\stackrel{0}{0}}$ \\
\hline న్స & 8 & $\ddot{-}$ & $\stackrel{\ddot{O}}{-1}$ & 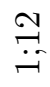 & $\stackrel{10}{\dddot{10}}$ & $\stackrel{\widetilde{N}}{\sim}$ & 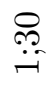 & $\stackrel{\circ}{\stackrel{+}{\pi}}$ & $\ddot{\sim}$ & $\begin{array}{l}\stackrel{H}{N} \\
\ddot{N}\end{array}$ & $\stackrel{8}{\dddot{N}}$ & $\ddot{\leftrightarrow}$ \\
\hline
\end{tabular}




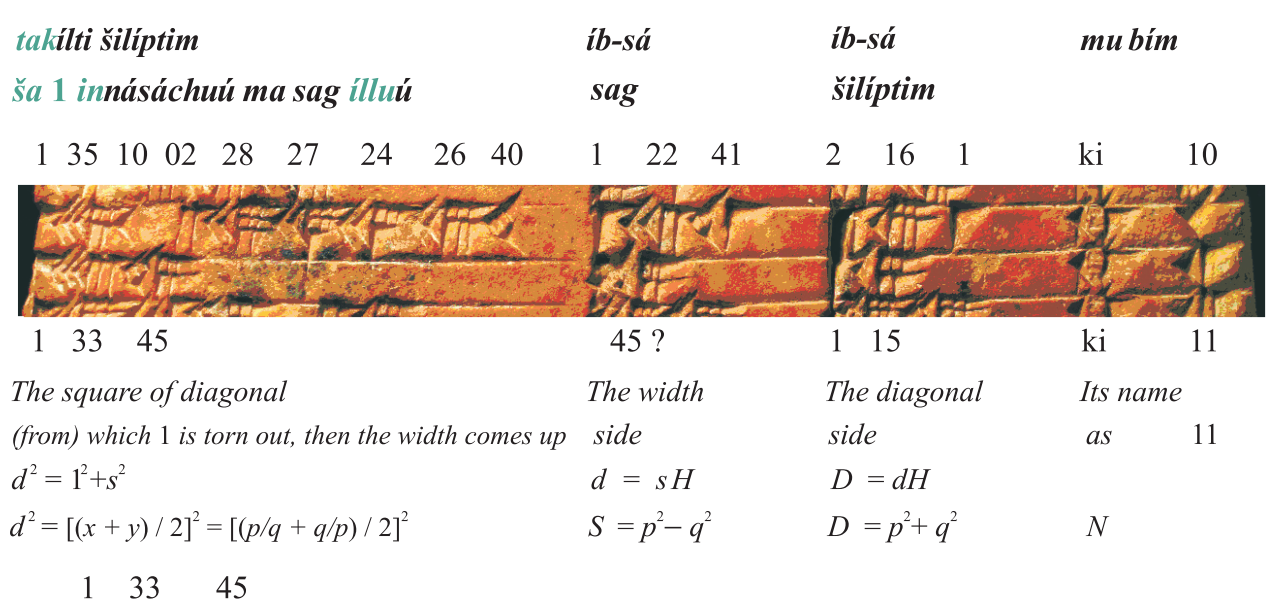

The row with the ordering number $N=11$

The row with the ordering number $N=12$

Figure 5. A part of the clay tablet Plimpton 322 with a cuneiform text. Headings above (in Sumerian-Akkadian transcription) and bellow (in English) the four columns of sexagesimal numbers explain their meaning in the selected rows with the ordering numbers $N=10$ and 11 . As $s, d, h=1$ are denoted sides of unit triangles and $S, D, H$ are integer sides of similar triangles. Also a detail of the tablet with the broken left edge and the discussed number 1 in the 12th row is included. (Both the images are cut off from the photos taken by Christine Proust [11.)

- By a direct multiplication $s^{2}=s \times s$ or $d^{2}=d \times d$ of simple two digit widths $s:(0 ; 11, \ldots, 1 ; 20)$ or diagonals $d:(1 ; 01, \ldots, 1 ; 40)$. In simple cases also the Old Babylonian special table of squares (IM 96183, Friberg (2007), [9. Appendix 7]) or combined multiplication table (Friberg (2007), [9. Appendix 2]) could have been used.

- By a parallel factorization of multi-digit sides $s, d, h$ of unit triangle: A multi-digit number can be decomposed into a product of smaller, ideally singledigit numbers. Then a multiplication by such a number can be performed as a succession of simple steps. The decomposition of n-digit sexagesimal fractional number $s=0 ; s_{1} s_{2} \ldots s_{n-1} \quad s_{n}$ can utilize that the last digit $s_{n}=f_{n} g_{n} \times 60^{-n}$ can be written as a product of the greatest divisor $g_{n}$ and a factor $f_{n}$. Multiplying the number

$$
s=0 ; s_{1} s_{2} \ldots s_{n-1} s_{n}=0 ; s_{1} s_{2} \ldots s_{n-1}+f_{n} g_{n} 60^{-n}
$$

by the reciprocal of the greatest divisor $h_{n}=100 ; / g_{n}$ causes that the last fraction disappears. It changes to $f_{n} \times 60^{-(n-1)}$. In this way, the resulting number 
$s \times h_{n}$ reduces to $n-1$ fractional digits. Thus repeating the multiplication by the reciprocals $h_{n-1}, \ldots, h_{1}$ of the last greatest divisors $g_{n-1}, \ldots, g_{1}$, the final (least) factor

$$
S=s \times h_{n} \times \cdots \times h_{1}=s \times H_{s}
$$

will be obtained, where the multiplier $H_{s}$ and its reciprocal $1 / H_{s}$ are determined by the relations

and

$$
H_{s}=h_{n} \times \cdots \times h_{1}=\left(1 / g_{n}\right) \times \cdots \times\left(1 / g_{1}\right) \times(100 ;)^{n}
$$

$$
1 / H_{s}=g_{n} \times \cdots \times g_{1} \times(100 ;)^{-n} .
$$

As $g_{n} \times h_{n}=100$; the reciprocals $g_{n}, h_{n}$ are single-digit sexagesimals. Then according to (8) and (9), the inequality $S \geq s$ is satisfied for the absolute value of the final factor $S$ and the original number $s$. Paradoxically, for their relative values, the opposite expression is valid. Evidently, in accord with (9)

$$
S=s H_{s} \leq 0 ; s_{1} \ldots s_{n} \times(100 ;)^{n}=s_{1} \ldots s_{n} .
$$

Friberg calls the factorization method: the trailing part algorithm and the final factor $S$ : the factor-reduced core. (Friberg (2007), [9]).

By this factorization method, the n-digit number $s$ can be reduced to the final factor (8): $S=s \times H_{s}$ and inversely, it can be decomposed to the product $s=S \times\left(1 / H_{s}\right)$.

The factorization can simplify calculation of squares of many-digit-numbers $s$ :

$$
s^{2}=s \times s=S^{2} \times\left(1 / H_{s}\right) \times\left(1 / H_{s}\right) .
$$

Inversely, by factorization of a square $s^{2}$ to the reduced factor $S^{2}=s^{2} \times\left(H_{s} \times H_{s}\right)$, supposing that its square root $S$ is known, the square root $s=S \times\left(1 / H_{s}\right)$ of the number $s^{2}$ can be calculated (F r i berg (2007), [9]).

It should be mentioned that Old Babylonian scribe had calculated with numbers in their relative form without the floating point therefore he had to keep the factors $(100 ;)^{n}$ or $(100 ;)^{-n}$ from relations (9) (more precisely, an idea of the absolute value of the calculated numbers) in his mind. It could easily lead to errors.

In technical praxis, Old Babylonians had to deal with both small and large objects. It is illustrated by their length unit system, inherited after Sumers (F r i ber g (2007), 9]): 
RUDOLF HAJOSSY

\begin{tabular}{|c|c|c|c|c|c|c|c|}
\hline Relations & \multicolumn{2}{|c|}{$6 \check{s} e=1 \check{s} u s i$} & \multicolumn{2}{|c|}{12 kúš $=1$ ninda } & \multicolumn{2}{|c|}{6 éše $=1$ us } & \\
\hline \multirow[t]{3}{*}{ Units } & $1 \check{s} e$ & 1 šusi & 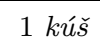 & 1 ninda & 1 éše & $1 u \check{s}$ & 1 danna \\
\hline & $\begin{array}{c}\text { grain } \\
\text { of barley }\end{array}$ & finger & cubit & reed & rope & length & distance \\
\hline & $\begin{array}{l}\approx 1 / 360 \mathrm{~m} \\
=2.78 \mathrm{~mm}\end{array}$ & $\begin{array}{r}1 / 60 \mathrm{~m} \\
16.7 \mathrm{~mm} \\
\end{array}$ & $1 / 2 \mathrm{~m}$ & $6 \mathrm{~m}$ & $60 \mathrm{~m}$ & $360 \mathrm{~m}$ & $10,800 \mathrm{~m}$ \\
\hline Relations & & \multicolumn{2}{|c|}{30 šusi $=1$ kúš } & \multicolumn{2}{|c|}{10 ninda $=1$ éše } & \multicolumn{2}{|c|}{$30 u \check{s}=1$ danna } \\
\hline
\end{tabular}

This system of different units assisted them in removing ambiguity of the relative form of inscription of sexagesimal numbers (without a boundary between their integer and fractional part). Although calculations were done with relative numbers, Old Babylonians were always interested in the absolute value of results: It mattered whether a purchased field had a width 130 ninda $(540 \mathrm{~m})$ or $1 ; 30$ ninda $(=18$ kúš $=9 \mathrm{~m})$.

The factorization formula (11) can be used independently for a calculation of squares of width $s^{2}=s \times s=S^{2} \times\left(1 / H_{s}\right) \times\left(1 / H_{s}\right)$ and also diagonal $d^{2}=d \times d=D^{2} \times\left(1 / H_{d}\right) \times\left(1 / H_{d}\right)$, where the multipliers $H_{s}, H_{d}$ are products of reciprocals from the greatest divisors of the last digits determined independently for the side $s$ and $d$, respectively. However, to obtain Tab. 2, only the common greatest divisors of the last digits of the both sides $s$ and $d$ have been used. Therefore, the multipliers $H_{s}=H_{d}=H$ have the same value and the final factors $S=s H, D=d H, H=h H$ in Tab. 2 can be interpreted as integer sides of triangles which are similar to the original unit triangles $s, d, h=1$.

This parallel factorization of sides $s$ and $d$ has an advantage against an independent factorization of the sides: the result is simpler (only one multiplier $H$ against two $H_{s}, H_{d}$ ). It can accelerate calculations (performed by one scribe) and save a space in a table of final results. (A similar space saving effect has a replacement of the trivial side $h=1$ of a unit triangle by the integer side $H$ of a similar triangle in Tab. 2.)

For simplicity, only the squares $d^{2}$ are shown in Tab. 2 as the squares $s^{2}=$ $d^{2}-1$ differ from $d^{2}$ only by the initial number 1 . Moreover, the square $d^{2}$ is always unambiguous but not $s^{2}$ as it can be seen in the second row of Tab. 2 where $d^{2}=1 ; 0015005615$. Then $s^{2}=0 ; 0015005615$. Relative values of these parameters 10015005615 and 15005615 , used by ancient scribes, illustrate that the initial digit 1 in $d^{2}$ does show where the missing floating point should be placed but the missing beginning zeros in $s^{2}$ make its absolute value uncertain. The statement is justified by new high-quality pictures of P322 made by Christine Proust (Fig. 1 and 5) published also in her online article [11. Her picture has definitely solved a long lasting dispute on the " $s^{2}$ or $d^{2}$ ?". In the 12 th row with $d^{2}=1 ; 2921 \ldots$ in Fig. 1.B and 5, the sequence: the numeral 1 - blank space - the numeral 29 can be seen. In the case of $s^{2}=0 ; 2921 \ldots$, the numeral 29 ought to be seen just at the vertical line parting the columns. (All numbers in P322 start at the parting lines!) The free space and the numbers 33 or 35 can be also clearly seen in the rows 11th or 10th in Fig. 5, respectively. (The old black-and-white picture of P322 in the 


\section{PLIMPTON 322: A UNIVERSAL CUNEIFORM TABLE}

Ne u g e bauer and S a chs primary article has unreadable left broken edge [1], 8]. So readers were obliged to believe the lucky persons who had opportunity to see the original tablet P322.)

As it follows from Tab. 2, the triangles for the simplest integers $x=2$ and 3 are similar to the well-known triangle with integer sides 3,4,5 used for delineation of perpendiculars in ancient building or surveying. The two triangles are only mutually turned. In accord with Fig. 3 , the number $x=2$ provides a steep triangle $(s=0 ; 45<1)$ while the number 3 corresponds to a gentle slope $(s=1 ; 20>1)$.

The exact boundary between the steep and mild slope of diagonal is determined by the diagonal in the unit square, with its unit width and length $(s=h=1)$ or by the square of the diagonal $d^{2}=1^{2}+1^{2}=2$. The Tab. 2 shows that the boundary lies between the row $N=9$ with the bold number $x=\mathbf{2} ; \mathbf{2 4}$ and the row $N=10$ with the magenta number $x=2 ; 30$. The latter allows to replace the exact condition $(0<s<1)$ for the width of steep triangle by a simpler, though less precise condition $(1<x<2 ; 30)$ for the generating number $x$. (Thus, when selecting $x$, the last condition allows to decide without further computation, whether a calculated triangle will be steep or mild. It certainly saves useless calculations and time to compile the table.)

The least steep diagonal $\left(d^{2}=1 ; 590015\right)$ in Tab. 2, close to the diagonal of the square $\left(d^{2}=2\right)$ is in the ninth triangle $(N=9)$, calculated for the number $x=2 ; 24$. The steepest diagonal $\left(d^{2}=1 ;\right)$ is in the first $(N=1)$ of the triangles in Tab. 2 , with a zero width $(s=0)$ calculated for $x=1$. Such a "triangle" was certainly a mystery for Old Babylonians. Everything depended on their interpretation of zero: Whether it was something awfully small (almost vertical segment in Fig. 3) or something non-existent - "nu" (not in Sumerian) (Neugebauer [15, Glossar p. 30]). On the other hand, Tab. 2 is mainly a table of square roots. From this point of view, the value zero or one of a root is not so puzzling.

Interest in steep triangles is natural, because their widths $s$ have the upper and lower limit $(0<s<1)$. The limits are also in the corresponding numbers $1<x<2 ; 30$. Triangles with a mild slope are limited only from their lower side by the intervals $1<s$ and $2 ; 30<x$, so an upper limit of the number $x$ is not known in advance. It will lead to many useless calculations. It is sufficient to determine only steep triangles since mild ones can be obtained, in accordance with Fig. 3, by a simple turning of the steep triangles.

The sides $s, d, h=1$ of calculated unit triangles in Tab. 2 were of interest to builders. Old Babylonian builders did not know an angle as an arc of unit circle but they could measure it as a side of unit triangle. They measured the slope of walls or embankments as a deviation $s$ from the vertical or horizontal line in the unit length $h=1$ kúš (Fig. 6). 

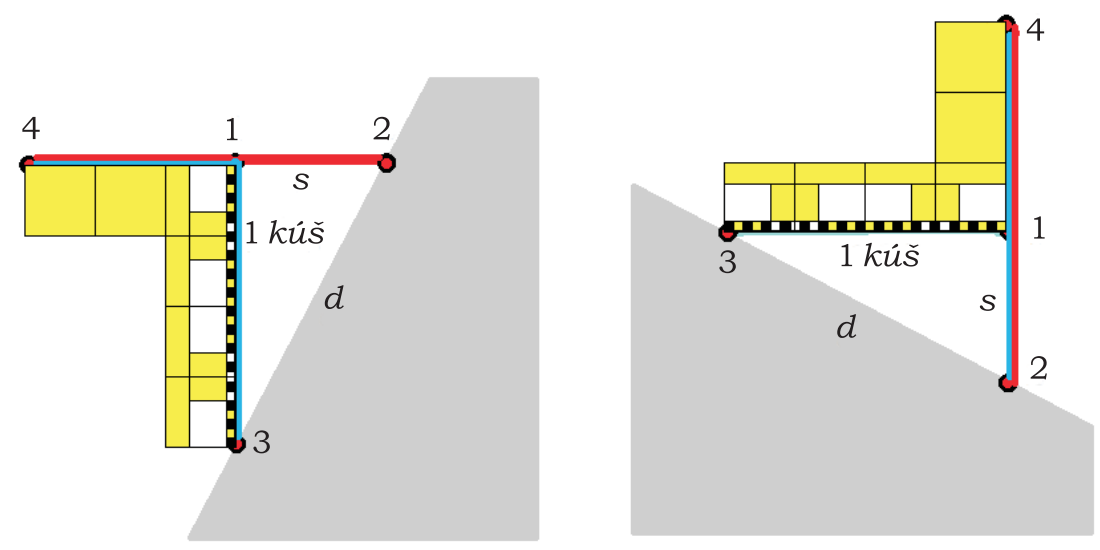

Figure 6. Expected measurements of a slope of a steep wall or a mild embankment by a suitable unit (white) triangle with the sides: width $(\operatorname{sag}) s$, diagonal $d$ and length $(u \check{s}) h=1$ cubit (kús).

A suitably orientated right angle is created by the yellow ruler with the scale

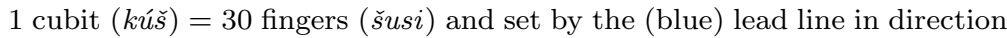
$\underline{13}$ or $\underline{42}$. A slope (šágal) determined by the width $s(\underline{12})$ is measured by the red line in direction $\underline{42}$.

A slope (šágal in Sumerian) represents a ratio between the width $s$ and the length $h$ of a right-angled triangle (Friberg: 44 p. 311]). To determine the inclination of the wall (diagonal $d$ ) in Fig. 6, the ancient builder would have used the formulation:

ina uš 1 kúš sag 16 šusi šágal=

$$
\text { in the length } 1 \text { cubit the width } 16 \text { fingers (is) the slope }
$$

It is in agreement with the tablet $Y B C 4673$, Robson [7, p. 183] and N e u ge bauer [15, Glossar p. 32, 12]. Since the width $s$ can serve as an inclination rate, the formulation is also included in the headings of the column $s$ in Tab. 2.

The width $s$ of a unit triangle changes in Tab. 2 continuously with the slope of diagonal $d$ therefore it is suitable for measurements in building and surveying. Just opposite, the similar triangles with integer sides $S, D, H$ change erratically. So they cannot be applied for measurements (with the exception of the well-known triplet $3,4,5)$. But they can help, using the relation (11), to square the sides of a similar unit triangle.

Because the length 1 kús (cubit) is small (around 1/2 m), the measuring unit triangles (e.g., 0;45, 1;15, 1;) in kúš do not guarantee a sufficient accuracy of the inclination measurements in surveying. A higher precision can be achieved by a similar sixty times $(100 ; \times)$ larger triangles with sides $(45 ; 115 ; 100 ;) k u ́ s ̌ s$ where $100 ; k u ́ s ̌ s 30 \mathrm{~m}$. 


\section{How the tablet Plimpton 322 was compiled?}

\section{Extension of The standard table of reciprocals}

The tablet P322 comprises 15 steep triangles but only 2 of them (1th and 11th) are also in Tab. 2. It means that the author of P322 wanted to generate a more detailed table of the triangles (rectangles) than the Tab. 2 which had been calculated using only the reciprocal pairs from The standard table of reciprocals (Tab. 1). To obtain further steep triangles, the author of P322 had to extend The standard table by new reciprocal pairs $x, y$ with numbers $x$ from the interval $1 \leq x<2 ; 30$, as it has followed from Tab. 2. Such numbers $x=p / q>1$ are produced by division of a greater number $p$ by a smaller $q$. (In today's terms, numbers $x$ represent improper fractions with $p>q$.) Furthermore, dividing one numerator $p$ by different denominators $q$, several fractions $x=p / q$ and consequently several triangles can be determined.

To minimize a computational time, it was necessary to choose the simplest, single-digit denominator $1 \leq q<100$;. (Of course, the numerator $p$ might be also two-digital.) If the simple numbers $p, q$ were from The standard table then a calculation of the improper fraction $x=p / q$ and its reciprocal $y=q / p$ was within a scribe routine.

The standard table (Tab. 1) shows that the denominator $q$ has 25 values $(1,2, \ldots, 54)$ and the numerator $p$ could have 40 figures $(1,2, \ldots, 4000 ;)$. From these figures, $25 \times 40=1000$ fractions can be assembled, including $(40-1)+\cdots+(40-25)=(39+15) \times 25 / 2=\mathbf{6 7 5}$ improper fractions $x=p / q>1$.

If a scribe calculated the fractions $x$ for a given denominator $q$ and gradually increased numerators $p>q$ until he found an invalid fraction $x \geq 2 ; 30$, then all his results, except the last one, were correct. However, many of the correct results $(x<2 ; 30)$ were calculated in vain, because they were equal to the results already obtained before, for the other (smaller) values of denominators $q$. Namely, a gradual increase of denominator $q, 2 q, 3 q, 4 q, 5 q, \ldots$ leads to an independent increase of numerator $p, 2 p, 3 p, 4 p, 5 p, \ldots$ but the value of a fraction $x=p / q=2 p / 2 q=3 p / 3 q=5 p / 5 q$ does not change by this proportional increase of the numerator and denominator. To avoid the useless calculation of fractions $x$ with the same value, it is necessary to exclude the numerators and denominators with a common divisors 2, 3 or 5. (Finding a common divisor was for a scribe a known operation, used also at the parallel factorization of sides of a unit triangle.)

The gradual elimination of unsuitable pairs $p, q$ with a common divisor and the subsequent calculation of fractions from suitable pairs until the first invalid fraction is found $x \geq 2 ; 30$ save a time and material. (The elimination of a fraction is faster than its calculation. Moreover, excluded or invalid fractions need not 
be stored for further processing.) The numerator $p_{t h}$ of the first invalid fraction $x=p_{t h} / q \geq 2 ; 30$ represents the threshold numerator for a given denominator $q$. (All fractions $p / q \geq p_{t h} / q$ are invalid as they give mild triangles.)

Calculation of improper fractions using criteria of the common divisor and slightly modified invalid fraction $x \geq 2 ; 25$ was first done by de Solla Price [3]. Friberg [4] used the condition $x \leq 4$. The both authors used, instead of numbers $p, q$ from The standard table, all regular numbers $p \leq 215$; and $q<100$; including the numbers 1 36, 148, 2 05, 2 08, 215 which were not in Tab. 1. (The regular sexagesimal number $x$ has a finite reciprocal number $1 / x$. Such number can be written in the form $x=2^{a} 3^{b} 5^{c}$ where exponents $a, b, c$ are integers. The numbers in Tab. 1 are regular.) However, apart from the 205 , the additional numbers are eliminated as unsuitable for calculation of improper fractions. So they do not influence the final extension of The standard table of reciprocals Tab. 3 .

A disadvantage of the criterion of invalid fraction $x \geq 2 ; 30$ is in wasting time with calculation of the invalid fraction and in the useless elimination of unsuitable pairs $p, q$ before the threshold numerator $p_{t h}$ is reached. The useless operations can be avoided when the criterion $x \geq 2 ; 30$ is replaced with a condition for the maximum appropriate fraction $x_{\text {max }}$ :

$$
x=p / q<x_{\max }=p_{\max } / q=2 ; 30 .
$$

Moreover, the last condition can be substituted for simpler criterion for the maximum numerator

$$
p_{\max }=2 ; 30 q>p>q .
$$

The maximum numerator $p_{\max }$ can be calculated in advance, so the eliminations of unsuitable pairs $p, q$ and calculation of searched fractions $x$ stop below the maximum value $p_{\max }$. There is no need to continue the elimination till the threshold numerator $p_{t h}>p_{\max }$ will be reached. (A modified criterion of the maximum numerator $p<2 ; 25 q$ was used by Friberg [9] and also suggested by Abdulaziz [13].)

Eventually, the numbers $x=p / q$, calculated from single-digit denominators $q$ and numerators $p$ from the interval (12), are shown in Tab. 3. The pairs $p, q$ themselves are from The standard table (Tab. 1). (Only the magenta numerators $p=1$; and 205; in the first and the last row of Tab. 3 have been determined additionally in an effort to find fractions $x$ with extreme numerators $p$.) Sorting of all calculated fractions $x$ in decreasing order gives them the order number $N$.

According to Tab. 3 , some of denominators $q(6,10,30,36,48)$ produced no fraction $x$. Also the greatest denominator $q=54$ failed as 25 possible numerators $(p>54)$ from The standard table (Tab. 1) had to be eliminated. In an attempt to obtain the widest possible spectrum of triangles, the author of P322 tried to find a numerator beyond Tab. 1 which could not be eliminated. The numerator $p$ cannot have a common divisor with the denominator $q=54=2.3^{3}$. Only such numerators are the multiples 


\section{PLIMPTON 322: A UNIVERSAL CUNEIFORM TABLE}

TABLE 3. The extended table of reciprocal pairs: (igi) $x=p / q \geq 1$ and (igibi) $y=q / p$ for a calculation of "all" steep right triangles where the pair $p, q$ is from The standard table (Tab. 1) and has no common divisor 2,3 or 5 , moreover, $1 ; \leq q \leq 54$; and $q<p<p \max =2 ; 30 q$. Sorting of all 39 calculated fractions $x$ in decreasing order gives them the order number $N$.

\begin{tabular}{|c|c|c|c|c|c|c|c|c|}
\hline$q$ & $\begin{array}{l}p_{\max } \\
2 ; 30 q\end{array}$ & $N_{235}$ & $p$ & $\begin{array}{c}\boldsymbol{x}=\boldsymbol{p} / \boldsymbol{q} \\
i g i\end{array}$ & $\begin{array}{c}\boldsymbol{y}=\boldsymbol{q} / \boldsymbol{p} \\
\text { igibi }\end{array}$ & $N_{t h}$ & $p_{t h}$ & $\begin{array}{c}\mathbf{N} \\
m u \text { bim }\end{array}$ \\
\hline \multirow[t]{2}{*}{$1 ;$} & $2 ; 30$ & 0 & $1 ;$ & $1 ;$ & $1 ;$ & & & 39 \\
\hline & & & 2 & 2 & $0 ; 30$ & 1 & 3 & 11 \\
\hline 2 & 5 & 1 & $3 ;$ & $1 ; 30$ & $0 ; 40$ & 2 & 5 & 22 \\
\hline \multirow[t]{2}{*}{3} & $7 ; 30$ & 1 & 4 & $1 ; 20$ & $0 ; 45$ & & & 29 \\
\hline & & & 5 & $1 ; 40$ & $0 ; 36$ & 1 & 8 & 18 \\
\hline \multirow[t]{2}{*}{$4 ;$} & 10 & 2 & 5 & $1 ; 15$ & $0 ; 48$ & & & 31 \\
\hline & & & $9 ;$ & $2 ; 15$ & $0 ; 2640$ & 3 & 15 & 5 \\
\hline \multirow[t]{4}{*}{5} & $12 ; 30$ & 1 & $6 ;$ & $1 ; 12$ & $0 ; 50$ & & & 32 \\
\hline & & & $8 ;$ & $1 ; 36$ & $0 ; 3730$ & & & 20 \\
\hline & & & $9 ;$ & $1 ; 48$ & $0 ; 3320$ & & & 15 \\
\hline & & & $12 ;$ & $2 ; 24$ & $0 ; 25$ & 2 & 16 & 1 \\
\hline $6 ;$ & 15 & 4 & - & - & - & 6 & 25 & - \\
\hline \multirow[t]{2}{*}{$8 ;$} & 20 & 4 & 9 & $1 ; 0730$ & $0 ; 5320$ & & & 34 \\
\hline & & & $15 ;$ & $1 ; 5230$ & $0 ; 32$ & 3 & 25 & 13 \\
\hline \multirow[t]{3}{*}{9} & $22 ; 30$ & 3 & 10 & $1 ; 06 \quad 40$ & $0 ; 54$ & & & 35 \\
\hline & & & $16 ;$ & $1 ; 4640$ & $0 ; 3345$ & & & 16 \\
\hline & & & 20 & $2 ; 13 \quad 20$ & $0 ; 27$ & 2 & 25 & 6 \\
\hline 10 & 25 & 5 & - & - & - & 2 & 27 & - \\
\hline 12 & 30 & 6 & $25 ;$ & $2 ; 05$ & $0 ; 2848$ & 33 & $(205 ;)$ & 9 \\
\hline \multirow[t]{2}{*}{15} & $37 ; 30$ & 7 & 16 ; & $1 ; 04$ & $0 ; 56 \quad 15$ & & & 37 \\
\hline & & & $32 ;$ & $2 ; 08$ & $0 ; 28 \quad 0730$ & 30 & $(2 \quad 08 ;)$ & 8 \\
\hline \multirow[t]{2}{*}{$16 ;$} & 40 & 6 & $25 ;$ & $1 ; 3345$ & $0 ; 38 \quad 24$ & & & 21 \\
\hline & & & 27 & $1 ; 4115$ & $0 ; 353320$ & 2 & 45 & 17 \\
\hline 18 & 45 & 7 & $25 ;$ & $1 ; 23 \quad 20$ & $0 ; 4312$ & 29 & $(2 \quad 05 ;)$ & 27 \\
\hline 20 & 50 & 8 & 27 & $1 ; 21$ & $0 ; 442640$ & 6 & 121 & 28 \\
\hline 24 & 100 & 9 & 25 & $1 ; 0230$ & $0 ; 5736$ & 25 & $(205 ;)$ & 38 \\
\hline \multirow[t]{5}{*}{25} & $102 ; 30$ & 5 & 27 & $1 ; 0448$ & $0 ; 553320$ & & & 36 \\
\hline & & & 32 & $1 ; 1648$ & $0 ; 465230$ & & & 30 \\
\hline & & & $36 ;$ & $1 ; 2624$ & $0 ; 4140$ & & & 24 \\
\hline & & & $48 ;$ & $1 ; 5512$ & $0 ; 3115$ & & & 12 \\
\hline & & & 54 & $2 ; 0936$ & $0 ; 274640$ & 4 & 121 & 7 \\
\hline \multirow[t]{4}{*}{27} & $107 ; 30$ & 6 & 32 & $1 ; 110640$ & $0 ; 503730$ & & & 33 \\
\hline & & & 40 & $1 ; 285320$ & $0 ; 4030$ & & & 23 \\
\hline & & & 50 & $1 ; 510640$ & $0 ; 32 \quad 24$ & & & 14 \\
\hline & & & 104 & $2 ; 221320$ & $0 ; 251845$ & 3 & 120 & 2 \\
\hline 30 & 115 & 10 & - & - & - & 22 & $(4000 ;)$ & - \\
\hline \multirow[t]{2}{*}{32} & 120 & 8 & 45 & $1 ; 242230$ & $0 ; 4240$ & & & 26 \\
\hline & & & 115 & $2 ; 203730$ & $0 ; 2536$ & 2 & $121 ;$ & 3 \\
\hline $36 ;$ & 130 & 11 & - & - & - & 19 & $(205 ;)$ & - \\
\hline 40 & 140 & 10 & $121 ;$ & $2 ; 0130$ & $0 ; 29374640$ & 18 & $(403 ;)$ & 10 \\
\hline 45 & $152 ; 30$ & 10 & 104 & $1 ; 25 \quad 20$ & $0 ; 42 \quad 11 \quad 15$ & 17 & $(208 ;)$ & 25 \\
\hline 48 & 200 & 10 & - & - & - & 17 & $(205 ;)$ & - \\
\hline 50 & 205 & 9 & $121 ;$ & $1 ; 3712$ & $0 ; 37 \quad 02 \quad 13 \quad 20$ & 16 & $\left(\begin{array}{ll}4 & 03 ;\end{array}\right)$ & 19 \\
\hline 54 & 215 & 9 & $205 ;$ & $2 ; 18 \quad 53 \quad 20$ & $0 ; 25 \quad 55 \quad 12$ & 16 & $(1025 ;)$ & 4 \\
\hline Total: & & 152 & & & & 281 & & \\
\hline
\end{tabular}


of the number 5 : $p=5,5^{2}=25,5^{3}=\mathbf{2} \mathbf{0 5}$; or $5^{4}=1025$;. Due to the additional condition (12) for the denominator $q=54$, only the numerators $54<p<215$; can occur. In this range, there is the only regular numerator $\boldsymbol{p}=5 \times 25=205$; with its finite reciprocal $\mathbf{1} / \boldsymbol{p}=(1 / 5)(1 / 25)=\mathbf{0} ; \mathbf{0 0 2 8} \mathbf{4 8}$. (Of course, the numerator 205 could be also found by a method of trials and errors.)

It is worthwhile to mention that the pair of the reciprocal (relative) numbers $5^{3}=\mathbf{2} \mathbf{0 5}$ and $12^{3}=\mathbf{2 8 4 8}$ served in the Old Babylonian tablets CBS 1215, CBS 10201 and $U M$ 29.13.21 from Nippur (F r i ber g [9]) as a base for calculation of new reciprocal pairs beyond the standard table. It is questionable whether the author of P322 knew these tablets or their copies.

Considering the divisors of numerators and denominators, the following threshold numerators $p_{t h}: 5^{3}=(\mathbf{2 0 5} ;), 3^{5}=(\mathbf{4 0 3} ;)$ or $2^{7}=(\mathbf{2 0 8} ;)$ have been calculated and shown in Tab. 3 .

The yellow denoted parameters in Tab. 3 help to compare calculation demands by the different criteria: the maximum numerator $p_{\max }$ or the threshold value $p_{t h}$. In this case

- $N_{235}$ is the number of eliminated fractions $x$, whose denominator $q$ and numerator $p$ (from the interval $q<p<p_{\max }=2 ; 30 q$ ) have a common divisor 2,3 or 5 ;

- $N_{t h}$ is the number of uselessly checked numerators $p$ from the interval $p_{\max } \leq p \leq p_{t h}$.

As it follows from Tab. 3 , the condition (12) for the maximum numerator $p_{\max }=$ $2 ; 30 q$ reduces the number 675 of improper fractions $x=p / q>1$ to $\mathbf{1 9 1}(=152+39)$, from which a checking of common divisors eliminates 152 and the remained 39 fractions $x$ can later on produce 39 steep triangles. A calculation of mild triangles should be more demanding because it would need to check not 191 but $484(=675-191)$ from the 675 possible fractions $x$.

Using the threshold numerator $p_{t h}$ as a criterion (de Solla Price [3], Friberg [4) increases, due to Tab. 3, the number of uselessly checked numerators $p$ by $\mathbf{2 8 1}$ and in vain calculated fractions $x$ (with the threshold numerators) by $\mathbf{1 4}$.

\section{Time and spatial demands of The extended tablet of reciprocals}

Table 3 enables to asses a time demand on its formation:

Calculation of 25 maximum numerators $p_{\max }=2 ; 30 q=2 q+q / 2$, election of 191 available numerators from the standard Tab. 1 and the intervals $q<p<p_{\max }$, exclusion of 152 pair $p, q$ with the common divisors 2,3 or 5 lasted (to the author R. H.) 80 minutes.

Calculation of 39 improper sexagesimal fractions $x=p / q$ lasted (without any calculator) 100 minutes. (For comparison, the same calculation with a simple calculator lasted only 8 minutes -13 times less.)

Manual ordering of 39 leaflets with the fraction values $x$ in decreasing order and their numbering lasted 15 minutes. 


\section{PLIMPTON 322: A UNIVERSAL CUNEIFORM TABLE}

Calculation of 39 reciprocals $y=q / p$ lasted 100 minutes.

An ancient scribe could be faster in multiplication but he had also to prepare recording material.

Preparation of 39 round handy tablets (bringing and shaping of a piece of clay) could take about $39 \times 5 \mathrm{~min} \approx \mathbf{2 0 0} \mathbf{~} \mathbf{m i n}$.

Thus the calculation and a temporal record of the data represented for one scribe around $\mathbf{5 0 0}$ min.

Eventually, for a clear arrangement and higher security of obtained results, it was necessary to rewrite them to a final tablet. It was sufficient to record 39 rows of the most important parameters $x, y, p, q, N$. A corresponding time assessment supposes knowledge of a recording speed (roughly, one edge per second) and a number of rewritten edges: According to Fig. 2, the smallest sexagesimal digit 1 has two edges while the greatest numeral 59 has them 28. In average, it is $\mathbf{1 5}$ edges per digit. Due to Tab. 3, the individual columns of parameters have following number of digits: $x(107), y(95), p(45), q(39), N(39)$. It is altogether 325 digits, $325 \times 15=4875$ edges. A transcript of the edges on a clay tablet then lasted around

$$
80 \min (=4875 \mathrm{sec} \times 1 \mathrm{~min} / 60 \mathrm{sec})
$$

If $\mathbf{2 0}$ minutes had been spent before preparing the final clay tablet and recording the headings above individual columns of parameters, then the total time for obtaining the final version of the extended table of reciprocals (Tab. 3) was approximately $600 \min =\mathbf{1 0}$ hours $=$ nearly $\mathbf{2}$ days of one scribe work.

As it has been already shown, the threshold numerator $p_{t h}$ criterion increases the number of uselessly checked numerators $p$ by $\mathbf{2 8 1}$ and in vain calculated fractions $x$ by 14 . Utilizing the previous time guesses, it will be found that the increased checking needs additional $281 \times 80 \mathrm{~min} / 152 \approx \mathbf{1 5 0} \mathrm{min}$ and the vain calculation will spend $14 \times 100 \mathrm{~min} / 39 \approx 36 \mathrm{~min}$. The less proper criterion protracts the time for obtaining The extended table of reciprocals (Tab. 3) for approximately $186 \mathrm{~min} \approx \mathbf{3}$ hours (in total, to $13 \mathrm{~h} \approx 2-3$ days). (In this case, the 25 maximum numerators $p_{\max }$ are not calculated but such saved up 4 minutes are negligible.)

Without any preliminary checking of common divisors, the shorter checking of $N_{235}=152$ improper fractions will be substituted by a longer calculation of the fractions. It means that 80 minutes of the checking will be replaced by $152 \times 100 / 39=$ 390 minutes of the calculations plus 100 minutes of additional computation of 39 invalid fractions $x \geq 2 ; 30$. It represents in total a protraction $410 \mathrm{~min} \approx 7 \mathrm{~h}$ in comparison to 10 hours in the case of the criterion of maximum numerators $p_{\max }=2 ; 30 q$. Thus, a complete construction of The extended table of reciprocals (Tab. 3) without preliminary checking will take approximately 17 hours $\approx \mathbf{3 - 4}$ days per a scribe. Of course, the construction could be accelerated by division of its calculations among a higher number of scribes. (A calculation by rude force [18, of all 675 improper fractions $x>1$, lasting $675 \times 100 / 39=1731 \mathrm{~min} \approx 29 \mathrm{~h} \approx 6$ days per a scribe seems improbable as it would increase gratuitously a possible 2-day-work to 8 days.) 
Spatial dimensions of the final version: According to Fig. 1.B and 5, the 21 places for digits, a character and gaps cover the width $127 \mathrm{~mm}$ of P322, then $6 \mathrm{~mm}$ is an average width of one place. 15 rows of a mathematical text and 3 rows of headings share the height of $88 \mathrm{~mm}$, then, $5 \mathrm{~mm}$ is their average height. A necessary dimension for a cuneiform text of the extended table (Tab. 3) is determined by the dimension of a digit: width $6 \mathrm{~mm} \times$ height $5 \mathrm{~mm}$; the maximum number of digits of individual parameters in Tab. 3: $x(4), y(4), p(2), q(1), N(2)$; the number of gaps (equal to one digit) between columns ( 4 at the 5 columns). In total, it is 17 digits along the width $17 \times 6 \mathrm{~mm}=\mathbf{1 0 2} \mathrm{mm}$. 39 rows of numbers and 3 rows of headings would represent the height $42 \times 5 \mathrm{~mm}=210 \mathrm{~mm}$ of one sided text or $\mathbf{1 0 5} \mathrm{mm}$ of the text on the obverse and reverse side of the tablet.

It is evident from the order numbers $N$ in Tab. 3 that The extended table with the ordered reciprocals had to be constructed en bloc as a complete unit of all 39 pairs $x, y$. Individual pairs in the ordered final table cannot be foreseen in advance (e.g., the first calculated pair: 1;0, 1; 0 is the last - 39th one in the ordered table while the last calculated pair: 2; 185320,$0 ; 255512$ in Tab. 3 is already the 4 th one).

\section{Calculations of a complete tablet P322}

Using the known reciprocal pairs $x, y$ from the ordered extended table Tab. 3 and applying the same method as in the case of the preliminary table of triangles Tab. 2, it is possible to make a more detailed table Tab. 4. Actual calculations can be performed in the following three steps:

1. By means of the reciprocal pairs $x, y$ from Tab. 3 , in agreement with the relations (5), the sides of a unit triangle $s=(x-y) / 2, d=(x+y) / 2$, $h=1$ are calculated.

2. From the sides $s, d, h$ of the unit triangle by a parallel factorization, the integer sides

$$
H=H h, \quad S=H s, \quad D=H d
$$

of a similar triangle are determined.

3. Eventually, from squares of the integer sides $S^{2}$ and the reciprocals $1 / H$, the squares of sides of the unit triangle

$$
s^{2}=S^{2}(1 / H)^{2}, \quad d^{2}=1^{2}+S^{2}(1 / H)^{2}
$$

are calculated.

During calculation, obtained parameters $s, d, h ; S, D, H ; S^{2}, 1 / H ; s^{2}, d^{2}, N$ have been temporarily registered on at least 8 individual tablets. (Initial parameters $p, q, x, y, N$ were written before on a special tablet, similar to Tab. 3.) The constant unit side $h=1$ was useless to register. From the squares $s^{2}, d^{2}$, 


\section{PLIMPTON 322: A UNIVERSAL CUNEIFORM TABLE}

it was sufficient to record the more unambiguous $d^{2}$. After getting all parameters for a given number $x$ (a row of the complete P322), for a transparency and security reasons, the results were transcribed to a separate, the most likely vertical tablet. Eventually, after a calculation of all $2 \times 39$ triangles it was supposed to rewrite the important results $s, d, H, d^{2}, S, D, N$ on a final (two-sided) tablet similar to Tab. 4. (The tablet P322 illustrates that already 15 rows, still incomplete half of all rows, have been rewritten on the obverse side of the horizontal tablet.)

The most important part of Tab. 4 is the triple of parameters $s, d, H$, probably located on the broken off part of the original complete tablet P322. From the triple, all remaining parameters could be determined: $x, y$ (by means of (4)); $S, D$ (by means of (13) ); $s^{2}, d^{2}$ (by means of (14)). Only initial pairs of numbers $p, q$ cannot be determined. They had to be laboriously compiled in Tab. 3. If there was a desire to made a universal table with all data interesting for practice but also with those strenuously obtained then the initializing pair $p, q$ could be placed on the left and right side of the tablet (similarly, as it is in Tab. 4).

Of course, a selection of parameters for the lost part of P322 is speculative but it should be done on a reasonable conjecture of the aim of the tablet. The initial words assign a similar problem in $M S$ 3502:

igi 2 uš sag ú šiliptum ennam

Number 2. What are the length, width and diagonal?

and the proper text presenting solution of the problem show that its significant parameters are $x, y, s, d, h=1, s^{2}, d^{2}$. It is natural that the missing yellow parameters occur in the supposed lost part of P322: $x, y, s, d$-in Friberg [9] and $s, d-$ -in Britton, Proust, Shni der [10]. A part of the parameters $S, D, H, 1 / H, S^{2}$ which might be used for the calculation of squares $s^{2}, d^{2}$ will still be missing in their contemplated complete tablet P322. Evidently, the most important of the 5 missing parameters is the length (multiplier) $H$. Without it, the sheer integer sides (the factorreduced core) $S, D$ are inutile as they are insufficient for a calculation of desired squares $s^{2}=S^{2}(1 / H)^{2}$ or $d^{2}=D^{2}(1 / H)^{2}$. (The calculation of $H=\sqrt{D^{2}-S^{2}}$ itself was beyond a common scribe knowledge. On the other hand, the calculation of $S=s H$ or $D=d H$ was within his ability.) For Friberg 9, the key parameter of P322 has been the square $d^{2}$ from which all remnant parameters could be determined by an independent factorization of squares $d^{2}$ and $s^{2}=d^{2}-1$, including the factors $H_{d}, H_{s}$. An inclusion of the pair $H_{d}, H_{s}$ together with proposed parameters $x, y, s, d$ would lead to an extremely large lost part of P322. However, by omitting the pair $H_{d}, H_{s}$, the factors $S, D$ have become inutile in P322. B ritton et al. [10] have considered the ordering $s, d, H, d^{2}, S, D$ unnatural. In contrary, we regard it as a short cut of the natural set of parameters $s, d, h=1, d^{2}, S, D, H, N$. 
A full agreement of the ordering numbers $N$ in the calculated tables Tab. 3, Tab. 4 and the real tablet P322 and a perfect accord of sexagesimal numbers in the corresponding data in Tab. 4 and P322, demonstrates a correct understanding of the technic by means of which the cuneiform tablet had been compiled. But it does not mean that the two tables (Tab. 4 and P322) have been obtained by an identical procedure.

For example, the author of P322 could avoid the parallel factorization of sides $s, d$ during calculation of the integer sides $S, D, H$. It was sufficient to realize that the reciprocal pairs

$$
x=p / q, \quad y=q / p
$$

represented fractions. Then a common fractional part of the sides

$$
s=(p / q-q / p) / 2 \text { and } d=(p / q+q / p) / 2
$$

was the factor

$$
1 / H=(1 / q)(1 / p)(1 / 2)
$$

To get rid of the common fractional part $1 / H$ in the sides $s, d, h$, it is enough to multiply the sides by the reciprocal value of the factor $1 / H$

$$
H=2 p q
$$

(The same effect is also provided by a factorization of the sides.) Usage of the multiplier (15) can strongly simplify the calculation of integer sides. They could be calculated independently. There would be no need to follow common divisors of the last fractional digits. It could accelerate such calculation and diminish the number of errors that could arise at a factorization.

\section{Short comments on the colors used in the Table 4:}

The yellow data are added to the existing extant of the damaged and uncomplete tablet P322.

The green data are unreadable in P322 extant.

The magenta data are correct but point out the errors made by ancient scribes in the real tablet.

The blue values $S, D, H$ of integer sides are obtained by factorization of simple, two-digit sides $s, d, h=1$ of unit triangles. In these cases instead of the blue factorized values $S, D, H$ shown in Tab. 4, the simple original values $s, d, h=1$ of the unit triangles are presented in the real tablet P322 and also used for a direct calculation of the square $d^{2}$. 
PLIMPTON 322: A UNIVERSAL CUNEIFORM TABLE

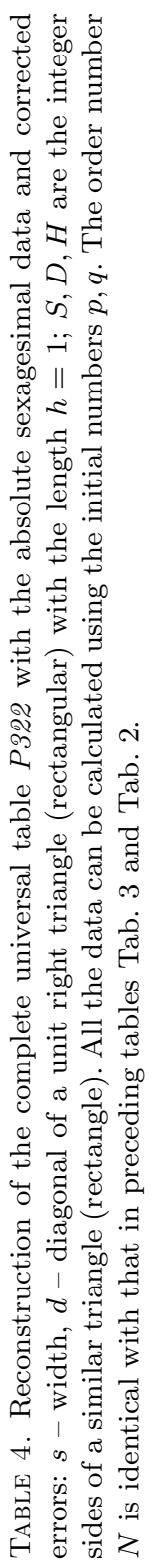

\begin{tabular}{|c|c|c|c|c|c|c|c|c|c|c|c|c|c|c|c|c|c|c|}
\hline \multirow{2}{*}{ 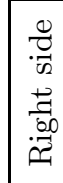 } & $\sigma$ & 10 & $\stackrel{N}{N}$ & ஸे & 10 & $\nabla$ & 0 & $\stackrel{12}{N}$ & 10 & $\stackrel{\sim}{\sim}$ & 아 & -15 & & $\infty \mid \hat{\curvearrowright}$ & & >|ות & $\stackrel{0}{0}$ & \\
\hline & 2 & $\stackrel{\mathcal{I}}{\mathcal{I}}$ & $\begin{array}{l}-1 \\
-1\end{array}$ & $\stackrel{20}{-1}$ & $\begin{array}{l}10 \\
\text { N } \\
\text { N }\end{array}$ & $\sigma$ & จ & 15 & ฉิ & $\stackrel{2}{\sim}$ & $\overrightarrow{-}$ & $N \propto$ & & 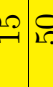 & & 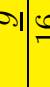 & $\stackrel{H}{2}$ & \\
\hline & Z & -1 & $\boldsymbol{N}$ & $\infty$ & 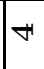 & 10 & 0 & $N$ & $\infty$ & 0 & $\stackrel{0}{-}$ & $=1$ & N) & 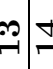 & & ك| & 1 & \\
\hline & 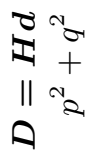 & $\mid \begin{array}{l}\stackrel{P}{+} \\
\sim \\
\sim\end{array}$ & 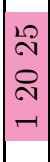 & $\mid \begin{array}{c}\stackrel{2}{+} \\
0 \\
20 \\
-1\end{array}$ & $\begin{array}{l}5 \\
8 \\
8 \\
10\end{array}$ & $\stackrel{\Lambda}{\infty}$ & $\begin{array}{l}-1 \\
\infty\end{array}$ & $\begin{array}{l}5 \\
0 \\
8 \\
8\end{array}$ & 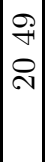 & $\left|\begin{array}{c}\stackrel{9}{+} \\
\stackrel{+}{-}\end{array}\right|$ & $\mid \begin{array}{l}0 \\
0 \\
0 \\
-1 \\
N\end{array}$ & & & 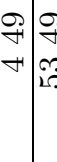 & 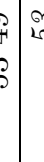 & ?al| & 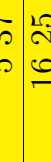 & \\
\hline & 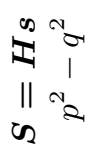 & {$\left[\begin{array}{l}8 \\
i s \\
-1\end{array}\right.$} & $\mid \begin{array}{l}1 \\
0 \\
0 \\
10\end{array}$ & $\mid \begin{array}{l}F \\
0 \\
-1 \\
-1\end{array}$ & $\begin{array}{l}\stackrel{9}{+} \\
\dot{m} \\
m\end{array}$ & 28 & $\stackrel{2}{20}$ & $\begin{array}{l}= \\
\infty \\
\infty\end{array}$ & $\begin{array}{l}\Omega \\
-\infty \\
-1\end{array}$ & $\begin{array}{l}-1 \\
\infty\end{array}$ & \begin{tabular}{l}
$F$ \\
\multirow{2}{*}{} \\
-1
\end{tabular} & & & 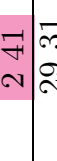 & $\begin{array}{lll}b & a \\
b & & \\
b & \end{array}$ & & $\begin{array}{ll}b & 0 \\
& i \\
v & 1\end{array}$ & \\
\hline & 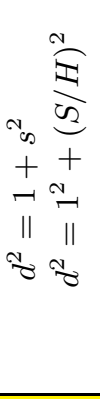 & $\begin{array}{r}20 \\
-1 \\
8 \\
0 \\
28 \\
-1 \\
-1 \\
\end{array}$ & $\begin{array}{l}10 \\
-1 \\
0 \\
8 \\
0 \\
10 \\
71 \\
-1 \\
\infty \\
10 \\
0 \\
10 \\
0 \\
10 \\
-1 \\
\end{array}$ & 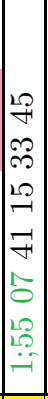 & 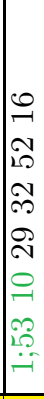 & 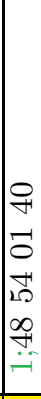 & 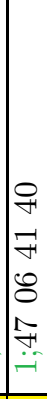 & 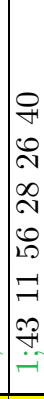 & 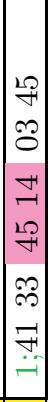 & 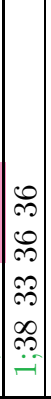 & 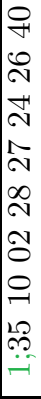 & 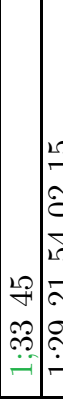 & 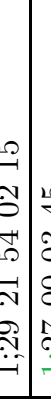 & 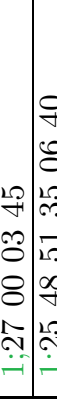 & 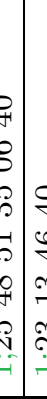 & 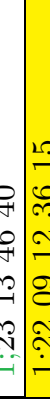 & 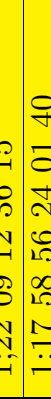 & \\
\hline & I $\frac{\tilde{s}}{\mathrm{~g}}$ & $\begin{array}{l}8 \\
\text { N }\end{array}$ & $\begin{array}{l}0 \\
\infty \\
1 \\
10\end{array}$ & 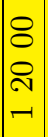 & $\begin{array}{l}8 \\
8 \\
20 \\
\sim \\
\infty\end{array}$ & $\stackrel{\mathcal{I}}{\beth}$ & $\begin{array}{l}8 \\
0 \\
0\end{array}$ & $\begin{array}{l}8 \\
8 \\
\text { \& }\end{array}$ & $\begin{array}{c}8 \\
0 \\
0 \\
-1\end{array}$ & $\begin{array}{l}8 \\
0 \\
-1\end{array}$ & $\begin{array}{l}8 \\
8 \\
\infty \\
+1 \\
-1\end{array}$ & $\forall$ & & 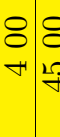 & $\begin{array}{l}8 \\
8 \\
8\end{array}$ & fil $\underset{\nabla}{\infty}$ & 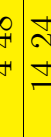 & \\
\hline & 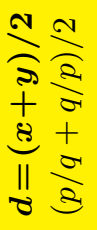 & 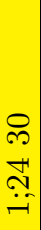 & 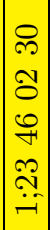 & $\begin{array}{c}\stackrel{2}{\sim} \\
\mathscr{8} \\
\approx \\
\dddot{\sim} \\
-1 \\
-1\end{array}$ & 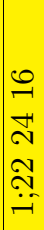 & 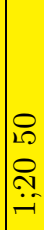 & 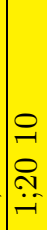 & 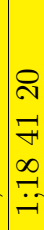 & \begin{tabular}{c}
12 \\
\hdashline \\
\hdashline \\
0 \\
$\infty$ \\
-1 \\
-1
\end{tabular} & $\mid \begin{array}{l}20 \\
0 \\
0 \\
-1 \\
-1\end{array}$ & 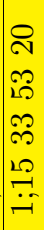 & 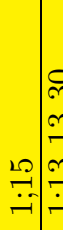 & 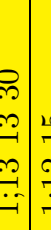 & 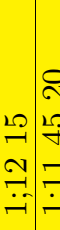 & 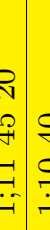 & 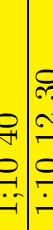 & 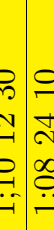 & \\
\hline $\begin{array}{l}0 \\
\frac{0}{0} \\
0 \\
0 \\
0 \\
0 \\
0 \\
0 \\
0\end{array}$ & 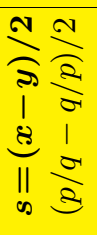 & $\mid \begin{array}{l}0 \\
\infty \\
0 \\
0 \\
?\end{array}$ & 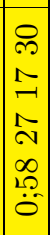 & 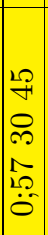 & $\begin{array}{l}5 \\
0 \\
8 \\
2 \\
0 \\
10 \\
0\end{array}$ & $\begin{array}{l}0 \\
0 \\
10 \\
0 \\
0\end{array}$ & $\mid \begin{array}{l}0 \\
0 \\
00 \\
i ? 0 \\
0\end{array}$ & $\begin{array}{l}\text { 와 } \\
\text { 내 } \\
0 \\
0 ? \\
0 \\
0\end{array}$ & $\mid \begin{array}{c}20 \\
6 \\
0 \\
20 \\
9 \\
+ \\
0 \\
0\end{array}$ & $\begin{array}{c}0 \\
8 \\
\infty \\
+ \\
0 \\
0\end{array}$ & $\begin{array}{l}8 \\
0 \\
10 \\
10 \\
10 \\
0 \\
0\end{array}$ & 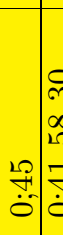 & 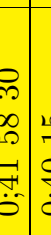 & 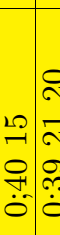 & 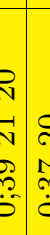 & 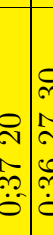 & 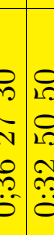 & \\
\hline
\end{tabular}




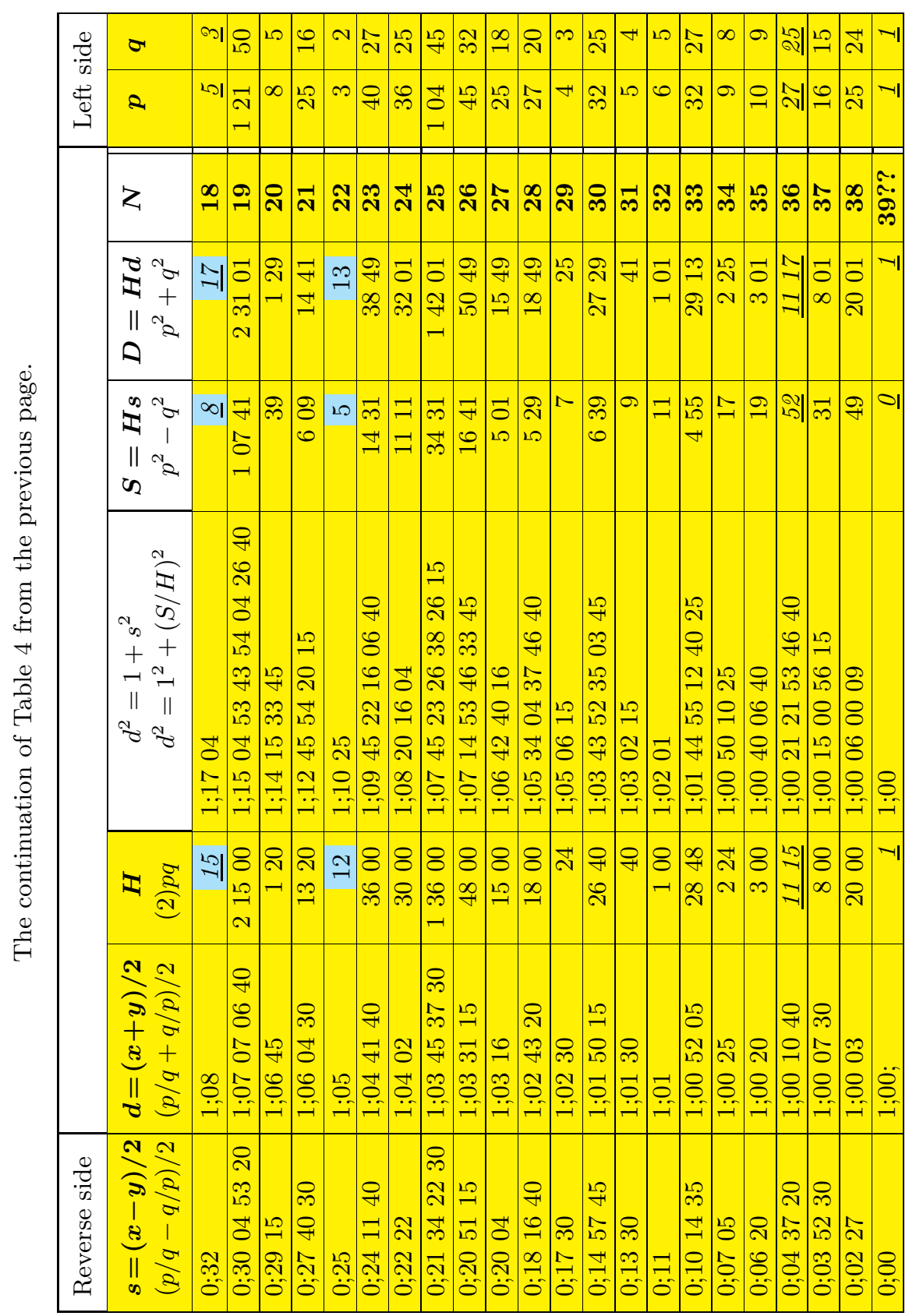




\section{PLIMPTON 322: A UNIVERSAL CUNEIFORM TABLE}

It is even less probable that the author of P322 was known with the relations between the triangle sides and the initial numbers $p, q$ which are today easy obtainable from the relations (5) and (13) by simple algebraic operations

$$
\begin{aligned}
s & =(x-y) / 2=(p / q-q / p) / 2=\left(p^{2}-q^{2}\right) /(2 p q), \\
d & =(x+y) / 2=(p / q+q / p) / 2=\left(p^{2}+q^{2}\right) /(2 p q), \\
h & =1 \\
H & =2 p q, S=p^{2}-q^{2}, D=p^{2}+q^{2} .
\end{aligned}
$$

When the initial numbers $p, q$ (underlined italics in Tab. 4) are odd, then the integer length is calculated as $H=p q$. The relations (16) are currently used for calculation of the "Pythagorean" triangles (triples). They were also used by N e u g e b a u e r and $\mathrm{S}$ a c h s [1] for proving a hypothesis that the tablet P322 has dealt with 15 Pythagorean triangles.

\section{Errors in the previous error analysis}

Comparing the numbers from Tab. 4 and from P322, it follows that Old Babylonians have made 6 errors in their tablet. (The original numbers of P322 can be found in Fig. 1. B or in the black-and-white photo of P322 in [8].) A position of the errors is denoted in Tab. 4 by the magenta color. Two of the errors are results of an insufficient attention during a transcription of data from an auxiliary to the final tablet:

- In the 9th row in the parameter $S$, there is, instead of the correct numeral 8, a similarly looking but incorrect digit 9 (Fig. 2).

- In the $\mathbf{2 n d}$ row in the parameter $\mathbf{d}^{\mathbf{2}}$, there is, instead of the correct pair of digits 50 06, an incorrect numeral 56. The error should be caused by a too small work tablet where the blank space representing zeros in the numbers 5006 was too small. So, two numbers 5006 have been misread as one number $\mathbf{5 6}$.

The remnant 4 errors are of an arithmetic character:

In spite of this, the mistakes are extremely useful since they prove that the data in P322 have been obtained in the order and way described above by the three steps.

The 1st error: In the 8th row in the parameter $d^{2}$, there is, instead of the correct pair of digits $45 \mathbf{1 4}$, an incorrect numeral $59(=45+14)$.

This parameter should be gained by the relation (14) as $d^{2}=1^{2}+S^{2}(1 / H)^{2}$, where values of the sides $S, H$ are provided by factorization of the sides $s, d$. 
The factorization has given for the 8th row in Tab. 4 values $S=1319$; and $H=1600=4 \times 4 \times 100$ and also $1 / H=0 ; 15 \times 0 ; 15 \times 0 ; 01=0 ; 000345$. Then

$$
\begin{aligned}
S^{2} \times(1 / H)^{2} & =(1319)^{2} \times(0 ; 15 \times 0 ; 15 \times 0 ; 01)^{2} \\
& =24615005615 \times\left(15 \times 0 ; 01^{6}\right)=0 ; 413345140345 .
\end{aligned}
$$

Eventually, the correct result for the square $d^{2}=1^{2}+0 ; 413345140345$, shown in Tab. 4, is obtained.

However, if the blank space (zero 00 ) in the last multiplication

$$
24615005615 \times\left(15 \times 0 ; 01^{6}\right)
$$

is overlooked, the telescoped number will be multiplied and it will lead to the faulty result

$$
s^{2}=246155615 \times\left(15 \times 0 ; 01^{6}\right)=0 ; 004133590345 .
$$

The whole calculation of P322 is made with the reciprocal pairs $x=p / q$ and $y=q / p$, therefore the product $x y=1 ; 00$ is exactly equal to the unit. According to (21), the unit had to be respected also in the calculation of the square $d^{2}=$ $1 ; 00+s^{2}$. So an incorrect result

$$
d^{2}=1 ; 00+0 ; 004133590345=1 ; 004133590345
$$

should be obtained.

As the ancient scribe made the calculations with relative sexagesimals, he obtained the result $d^{2}=1+4133590345=14133590345$, where he had done a second error - omitted the second (yellow) zero. Thus, due to the double error, he finally obtained the incorrect result written in P322.

Evidently, a calculation with relative numbers and blank spaces instead of zero had to be a frequent source of errors. However, the error connected with an incorrectly determined fractional part of the calculated number is done by nowadays authors, too:

Friberg [4, p. 297] calculated squares $s^{2}$ and $d^{2}$ directly as $S^{2}(1 / H)^{2}$ and $D^{2}(1 / H)^{2}$. After an omission of zero and calculation with relative numbers, he obtained the wanted results $s^{2}=4133590345$ and $d^{2}=14133590345$ which, as he stated, obey the condition $d^{2}=s^{2}+1$, so the incorrect results cannot be revealed. But respecting absolute values and the unit 1;00, he could reveal the mistake. He would have to calculate the values $s^{2}=0 ; 004133590345$ and $d^{2}=0 ; 014133590345$ which do not obey the condition $d^{2}=s^{2}+1 ; 00$.

Similarly, wanting to get the incorrect result $d^{2}=14133590345$ by the N e u g e ba uer and S a chs procedure (16), the authors (Britton et al., [10, p. 537] show at first the correct result $d^{2}=\left(p^{2}+q^{2}\right)^{2} /\left(4 p^{2} q^{2}\right)=$

$$
(7132001) \times(0 ; 0003305615) \times(0 ; 0004)=1 ; 413345140345
$$




\section{PLIMPTON 322: A UNIVERSAL CUNEIFORM TABLE}

for $p=32$ and $q=15$. However, if 7132001 is mistaken as 71321 then, according to the authors, the wanted incorrect result is obtained

$$
d^{2}=(71321) \times(0 ; 0003305615) \times(0 ; 0004)=1 ; 4133590345 .
$$

But they are wrong. They should have got the value $d^{2}=0 ; 014133590345$ which should warn them about a mistake, since a diagonal of a unit triangle must be greater than $1 ; 00$.

The discussed errors of the authors do not reduce the quality of their articles. They only document that who does numerical calculations he makes the numerical errors - today, just like 4,000 years ago.

The bad value of the square $d^{2}$ in the 8th row of P322, due to omission of zeros, can be obtained even by two other ways:

1. Bruins [17] started from the value $s=0 ; 495615$ (Tab. 4) of which square he counted directly as

$$
\begin{aligned}
s^{2} & =(0 ; 50-0 ; 000345)^{2} \\
& =0 ; 4140-0 ; 000615+0 ; 000000140345 \\
& =0 ; 413345140345 .
\end{aligned}
$$

If a scribe omitted one of the zeros in the third term and telescoped it to the value $0 ; 0000140345$, then he gained the wrong result $s^{2}=0 ; 4133590345$. This error would be transferred to square $d^{2}=1 ;+s^{2}$ as well.

2. Anagnostakis and Goldstein [14] applied the relations (7) between squares $s^{2}, d^{2}$ and reciprocal pairs $x, y$. The triangles in the 8th row of Tab. 4 have the ordering number $N=8$, for which Tab. 3 gives the values $x=2 ; 08$ and $y=0 ; 280730$. Then, according to (7)

$$
\begin{aligned}
d^{2} & =\left(2 ; 08^{2}+0 ; 280730^{2}\right) / 4+1 / 2 \\
& =(4 ; 3304+0 ; 1311005615) / 4+0 ; 30 \\
& =1 ; 413345140345 .
\end{aligned}
$$

If a scribe overlooked the zero in the second term and telescoped it to the value $0 ; 13115615$, then he got the wrong result $d^{2}=1 ; 4133590345$.

The incorrect value of square $d^{2}$ while the sides $S, D$ are correct in the 8 th row of P322, suggests that the squares $s^{2}, d^{2}$ were calculated either later than $S, D, H$ or independently: from the numbers $p, q$, reciprocal pairs $x, y$ or as a direct square of the side $s$. The reverse steps (from $d^{2}$ to $S, D$ ), promoted by F r i berg [9] and R o b s o n [7, should lead to wrong sides $S, D$, but those are, however, in the 8th row right. 
The 2nd error: In the 13th row in the parameter $S$, there is, instead of the correct number 241 , the incorrect value $71201=241^{2}$.

This error relates to the fact that for calculating a square $s^{2}=S^{2} /(1 / H)^{2}$ of a unit triangle, it is necessary to calculate and store a square $S^{2}$ of a similar integer triangle. During transcription of results from auxiliary tablets to the final tablet, the value $S^{2}$ was mistakenly written instead of $S$.

This error is explained similarly by Friberg [4] but Robson [7] and Britton et al. 10] suggest another explanation: They suppose that during a factorization instead of the sides $s, d$, the pair $s^{2}, d$ was mistakenly factorized. It should lead to the incorrect result $S^{2}, D$. Their conjecture is wrong. As it follows from (13), the parallel factorization is a linear process: the sides $s, d$ increase linearly to $S, D$. After such factorization, the pair $s^{2}, d$ enlarges to some value $S_{2}=H s^{2}$ and $D=H d$. The value $S_{2}=H s^{2}$ will differ from the wanted $S^{2}=H^{2} s^{2}$, shown in P322. (It is proven by direct calculations, too.) Also none of the other methods that explained successfully the previous error in the 2nd row (numbers $p, q$; reciprocal pairs $x, y$; direct square of $s$ ) can explain this error in the 13 th row.

The 3rd error: In the 2nd row in the parameter $\boldsymbol{D}$, there is, instead of the correct number $\mathbf{1 2 0 2 5}$, the incorrect value

$$
\mathbf{3 1 2 0 1 0 0 \approx 1 2 0 2 5 \times 1 2 \times 1 2 = 3 1 3 0 0 0 0 .}
$$

This error can be explained as a consequence of two mistakes: a different factorization of the initial sides $s=0 ; 58271730$ and $d=1 ; 23460230$ from Tab. 4 and a subsequent omission of zero during a calculation of the value $D=3130000$.

After two factorization steps, the initial (yellow) sides $s, d, h$ of a unit triangle changed to the values

$$
\begin{aligned}
0 ; 58271730 \times 2 \times 12=23 ; 2255, \\
1 ; 23460230 \times 2 \times 12=33 ; 3025, \\
1 \times 2 \times 12=24 .
\end{aligned}
$$

The last (green) numbers have a common divisor 5, and then in the third factorization step it was necessary to multiply by a reciprocal value of the divisor - by the number 12 . From some reason (darkness, a higher priority work, exchange of scribes), the third round of factorization was interrupted. Before the interruption, the calculator just managed to multiply the diagonal

$$
1 ; 23460230 \times 2 \times 12^{2}=642 ; 05 .
$$

Restoring the calculations (perhaps next day, with new tablets), the scribe continued to multiply by 12 . From the previous records, he found that the sides $s, h$ had already been multiplied by 12 (forgetting that it had been done in the second round). 


\section{PLIMPTON 322: A UNIVERSAL CUNEIFORM TABLE}

Thus, by mistake, instead of the sides $s, h$, he again multiplied the diagonal $d$. Thus, the third factorization round ended with the final triplet

$$
\begin{aligned}
0 ; 58271730 & \times 2 \times 12=23 ; 2255, \\
1 ; 23460230 & \times 2 \times 12^{3}=12025, \\
1 & \times 2 \times 12=24 .
\end{aligned}
$$

After another two rounds of a correct factorization of this incorrect triplet, he finally obtained for integer sides of a triangle the result

$$
\begin{aligned}
& S=0 ; 58271730 \times 2 \times 12^{3}=5607, \\
& \boldsymbol{D}=1 ; 23460230 \times 2 \times 12^{5}=\mathbf{3 1 3 0 0 0 0}, \\
& H=5736=1 \times 2 \times 12^{3}
\end{aligned}
$$

with the correct values of $S$ and $H$ shown in Tab. 4 and the incorrect value $\boldsymbol{D}=$ 3130000 slightly different from $D=3120100$ which is written in P322. The last erroneous value could occur again due to omission of the blank space (zero) during the fourth round of factorization:

$$
\begin{aligned}
D & =1 ; 23460230 \times 2 \times 12^{4} \times 12=160500 \times 12=(160000+500) \times 12 \\
& =3120000+10000=3130000-\text { a "correct" result in Tab. } 4 \\
& =3120000+100=3120100-\text { an "incorrect" result in P322. }
\end{aligned}
$$

A probability of this error is increased by the fact that it could be produced also by interruptions in the second or fourth round of factorization.

This erroneous diagonal $D$ was explained by Bruins [17] and later by Friberg [4], Robson [7, Britton et al. [10] as an excessive factorization made by a calculator who did not realize that he had already removed all fractional digits. It is implausible as he knew in advance how many fractional digits should be removed (only four in this case). Moreover, at the excessive factorization, the number 5 is no more a common divisor. Therefore its reciprocal 12 is no more suitable for such factorization. On the other hand, the incorrect relative value $D=31201$ is explained by them as a transcript mistake because the sexagesimal numerals 1201 and 13 can be with a little incaution easily confused.

The wrong diagonal $D$ suggests that the square $d^{2}$ of a unit triangle is determined using the equation (14) only from the square $S^{2}$ of integer triangle and not from $D^{2}$ because in the latter case the wrong value of $D$ would lead to a significant error in the calculated square $d^{2}$ in the 2nd row of P322. The wrong diagonal $D$ also shows that no accuracy checking of the calculated squares $d^{2}=s^{2}+1$; envisaged by Friberg [4], has been performed. (It is understandable. It would have significantly prolonged calculations.)

The wrong value of diagonal $D=3130000$ is $12^{2}$-times larger than the correct value 12025 . It suggests that the author of P322 did not know the relation (15) for the multiple $H=2 p q$ between the sides of unit and integer triangles. The relation could help him to get rid of errors connected with a factorization. 
Actually, due to Tab. 4, the numbers $p, q$ in the 2 nd row have values 104 and 27, respectively. Then $H=2 p q=2 \times 104 ; \times 27=2 \times 8^{2} \times 3^{3}=2 \times 12 \times 12^{2}=5736$. Evidently, using the multiple (15), the wrong result $D$ should preferably consist of the elementary multipliers $104,27,9,8,3$ but not of the numbers 12 or $12^{2}$ typical for factorization in this case.

The 4th error: In the 15th row in the parameter $S$, there is, instead of the correct number $\mathbf{2 8}$, the incorrect value $\mathbf{5 6}(=28 \times 2)$.

There is a general meaning ( $\mathrm{Bruins}$ [17, Friberg [4], Rob son [7], $\mathrm{Britt}$ on et al. [10]) that the error is a consequence of a different factorization of the individual sides $s=0 ; 3720$ and $d=1 ; 1040$ from Tab. 4. We suppose that it should be provoked by a time distress when the data of the last (15th) row were calculated in the last moment, just during transcription of results to the tablet P322. Such distress should require dividing the factorization between two scribes. The scribes factorized the sides $s$ and $d$ in the following two steps:

\begin{tabular}{r|r|l}
\hline \multicolumn{1}{c|}{ The first scribe } & \multicolumn{1}{|c|}{ The second scribe } & The comment \\
\hline $0 ; 3720 \times 3=1 ; 52$ & $1 ; 1040 \times 3=3 ; 32$ & 20 is a divisor and 3 its reciprocal. \\
$S=1 ; 52 \times 30=\mathbf{5 6}$ & $D=3 ; 32 \times 15=\mathbf{5 3}$ & 2 or 4 are divisors, \\
$H_{s}=3 \times 30=\mathbf{9 0}$ & $H_{d}=3 \times 15=\mathbf{4 5}$ & 30 and 15 their reciprocals. \\
$D_{s}=d H_{s}=\mathbf{1 4 6}$ & $S_{d}=s H_{d}=\mathbf{2 8}$ & \\
\hline
\end{tabular}

Evidently, the first scribe overlooked the divisor 4 for the last fractional digit 52 and obtained twice larger values $S=56$ and $H_{s}=90$ instead of 28 and 45 shown in Tab. 4 . Nevertheless, he could obtain the correct value of the square

$$
d^{2}=1 ;+S^{2}\left(1 / H_{s}\right)^{2}=1 ;+(56 \times 0 ; 20 \times 0 ; 02)^{2}=1 ; 23134640
$$

shown both in Tab. 4 and P322. The same correct value might be calculated also by the second scribe: $d^{2}=D^{2}\left(1 / H_{d}\right)^{2}=(53 \times 0 ; 20 \times 0 ; 04)^{2}=1 ; 23134640$. Of course, the values $S=\mathbf{5 6}$ and $D=\mathbf{5 3}$ in P322 do not correspond to the same integer triangle.

\section{Paradox of the tablet P322}

Just this opinion, that P322 is not the table of integer triangles (Pythagorean triplets) $S, D, H$ but only factors $S, D$ provided by independent factorizations of squares $s^{2}, d^{2}$ of sides of a unit triangle (rectangle), is hold by Friberg [9].

Paradoxically, Friberg in his earlier article ( 4, pp. 290-292]) still talked about Pythagorean triangles (triplets). He compiled the extended tablet P322 in agreement with the relations (5), (13), (14) and by application of the parallel factorization of sides $s, d$. He still considered errors in line 2 and 15 as a result of an incorrect parallel factorization. 


\section{PLIMPTON 322: A UNIVERSAL CUNEIFORM TABLE}

The key position of the squares $s^{2}, d^{2}$ in later Friberg's interpretation means that P322 should be completed just in an opposite way as in the case when it represented a table of triangles:

$$
\begin{aligned}
s^{2}, d^{2} \longrightarrow S^{2} & =s^{2} \times H_{s}^{2}, \quad D^{2}=d^{2} \times H_{d}^{2} \longrightarrow \\
\longrightarrow S & =\sqrt{S^{2}}, \quad H_{s}, \quad D=\sqrt{D^{2}}, \quad H_{d} \longrightarrow s=S / H_{s}, \quad d=D / H_{d} .
\end{aligned}
$$

The way of obtaining the squares $s^{2}, d^{2}$ is not discussed by him but there is a possibility to calculate them, due to (7), from reciprocal numbers $x, y$ as $\left(x^{2}+y^{2}\right) / 4 \pm 1 / 2$.

Individual members $S, D, H_{s}, 1 / H_{s}, H_{d}, 1 / H_{d}$ of the factorization are interesting in calculation of square roots of multidigital numbers $\left(s^{2}, d^{2}\right)$ which occur when quadratic problems are solved. Multidigital squares are decomposed into lessdigital multiples with easier computed roots. For example, in the line 10 in Tab. 4, the square $d^{2}$ is a 9-digit number; side $d$ has 5 digits; factorization terms $D, H$ - Friberg's reduced factorization cores - have 3 digits. (This Old Babylonian problem of multiplication of huge numbers by their decomposition to smaller multipliers has remained until now. Only today's computers with limited registers use fast, more sophisticated algorithms of decomposition, for example, K a r a t s u b a multiplication [19].)

In agreement with the procedure, F r i b e r g [9] proposes the following content of P322 extended by its lost (yellow) part:

$$
x, y, s, d, d^{2}, S, D, N .
$$

Factorized multiples $H_{s}, H_{d}$ are missing in the supposed table, though they would be more interesting for a table owner than the reciprocal pair $x, y$, which could be easily calculated as a sum or difference of the sides $d, s$ using the relation (3). Without the multiples $H_{s}, H_{d}$, the meaning of the reduced cores $S, D$ is doubtful because only a simultaneous knowledge of the multiples and values $S, D$ enables to calculate the sides $s, d$ or their squares $s^{2}, d^{2}$.

Friberg [9, p. 436] promotes factorization by squares. Thus the mistake in line 13, the number $S^{2}=71201$ in column 3 is a result of such factorization. Then, after Friberg: "the author of Plimpton 322 may have forgotten to compute the square side of 71201 . The correct entry in line 13 of column 2 would be $241(=\sqrt{71201})$." There is only a question whether such type of factorization was performed indeed. The discussion of the 1st error ( $d^{2}$ in 8th row) has shown that it was not.

According to Friberg [9], in the case of the independent factorization, the discussed data from the 2 nd and 15 th row are already no more errors. There is only a difference in the number of factorization steps: The square $s^{2}$ in the 2nd row is factorized four times with the overall multiple $H_{s}^{2}=2^{2} \times 12^{2} \times 12^{2} \times 12^{2}$, while the square of diagonal $d^{2}$ is factorized 6 times with the overall multiple $H_{d}^{2}=2^{2} \times 12^{2} \times 12^{2} \times 12^{2} \times 12^{2} \times 12^{2}$. According to F r i ber g [9, p. 436], this should be "similarly in the case of line 15", too. An independent factorization of squares $s^{2}$ and $d^{2}$ should give the entries $S=56$ and 
$D=53$ in the line 15 . We will show, however, that such factorization cannot give such numbers:

Evidently, in this case due to Tab. 4, the square $d^{2}=1 ; 23134640$. Then, square $s^{2}=d^{2}-1=0 ; 23134640$. The equal trail number $640=400=20^{2}$ has the reciprocal $3^{2}$. Then, from the initial squares after the first round of factorization the following numbers are obtained

$$
1 ; 23134640 \times 3^{2}=12 ; 2904 \text { and } 0 ; 23134640 \times 3^{2}=3 ; 2904 .
$$

Again the common new trail number $4=2^{2}$ has the reciprocal $30^{2}$. Then, after the second round of factorization, the numbers

$$
12 ; 2904 \times 30^{2}=30716=D^{2}
$$

and

$$
3 ; 2904 \times 30^{2}=5216=S^{2}
$$

are obtained. Their square roots give the factorization terms

$$
D=\mathbf{1 4 6}, \quad S=\mathbf{5 6}, \quad H_{d}=H_{s}=3 \times 30=\mathbf{1 3 0},
$$

in accord with the above discussed results of the first scribe for the line 15 . However, the "independent" factorizations of the squares $s^{2}, d^{2}$ fail to explain why only the value $D=146$ can be computed but not the correct value 53 , envisaged by Friberg [9] and found by the second scribe.

Of course, it is possible to obtain the value $D=53$, but it will need squares of the divisor 4 and his reciprocal 15 , used by the second scribe in the second round of factorization of the current numbers $1 ; 23134640 \times 3^{2}=12 ; 2904$ and $0 ; 23134640 \times 3^{2}=3 ; 2904$. It will need a new trail number $904=544=16 \times 34$ which contains the searched divisor $16=4^{2}$ with its reciprocal $15^{2}$. Then, after the second round of factorization, the numbers $12 ; 2904 \times 15^{2}=4649=D^{2}$ and $3 ; 2904 \times 15^{2}=1304=S^{2}$ are obtained. Their square roots give the results $D=\mathbf{5 3}, S=\mathbf{2 8}, H_{d}=H_{s}=3 \times 15=\mathbf{4 5}$, of the second scribe for the line 15 . The reproduction of the second scribe results is very artificial and depends on the previous knowledge, in contrast to a very natural procedure of the first scribe. So the independent factorizations of squares $s^{2}, d^{2}$ cannot explain the error in the line 15 because it will provide only results of the first scribe.

The observed equality of the multiples $H_{d}=H_{s}$ is no surprise. It could be expected even at the independent factorization of squares $s^{2}, d^{2}$ because the factorized fractional parts of these squares are exactly equal. Inequality of multiples $H_{d}, H_{s}$ occurs in P322 only in two cases (line 2 and 15). Therefore, it is plausible that the inequality of $H_{d}, H_{s}$ is a result of an accidental miscalculation and not of an intentionally independent factorization.

There is also an additional argument against the independent factorization of the squares $s^{2}, d^{2}$ : From the wrong $d^{2}$ in the lines 2 and 8 of P322, also incorrect values of parameters $S, D$ should be expected. However, three of them are correct and also the incorrect value of the fourth parameter ( $D$ in the line 2 ) has already been explained by a forced interruption of calculations and not as an intentional independent factorization. 


\section{PLIMPTON 322: A UNIVERSAL CUNEIFORM TABLE}

The width $S=56$ and diagonal $D=53$ could appear in the line 15 only when the sides $s, d$ themselves are independently factorized but not the squares $s^{2}, d^{2}$. Even the factorization of the sides $s, d$ cannot be considered intentionally independent. If it actually was, the author of $P 322$ ought to continue in factorization of the side $S=105$ in the 5 th row or of the sides $S=45, D=115$ in the line 11 of the P322.

Besides the qualitative difference between the triangle and factorization core interpretation of P322, there is also a paradoxical quantitative difference: From one point of view, the factorization enlarges fractional sides $s, d, h=1$ of a unit triangle $H$-times to the integer sides $S=s H, D=d H, H$ of a similar triangle. After Friberg [4, however, the same original sides $s=S \times 1 / H, d=D \times 1 / H$ are reduced $1 / H$ -times to the factorization cores $S, D$. For example, in the line 15 in Tab. 4, the sides $S=28, D=53$ are $3 \times 15=45$-times larger than the initial sides $s=0 ; 3720$ and $d=1 ; 1040$. On the other hand, according to $\mathrm{Fr}$ i b e r g [4, 9, the factorization cores $S=28, D=53$ are $20 \times 4=\mathbf{8 0}$-times smaller than the original side $s=3720$ and $d=11040$ expressed in relative numbers. Which of the contradictory statements is correct?

A brief mathematical answer to the question is given by the inequality (10). However, a deeper insight in the paradox is provided by the relation (2) between sides of a unit triangle $d^{2}-s^{2}=x y$. In the first case, a calculation with absolute values gives the result

$$
d^{2}-s^{2}=1 ; 1040^{2}-0 ; 3720^{2}=1 ; 23134640-0 ; 23134640=\mathbf{1} ; \mathbf{0 0} .
$$

In the second case, a calculation with the relative values gives

$$
11040^{2}-3720^{2}=123134640-23134640=1(00000000) .
$$

Since the tablet P322 has been obtained from reciprocal pairs $x=p / q$ and $y=q / p$, then their product $x y$ can only have one possible absolute value $\boldsymbol{x y}=\mathbf{1 ; 0 0}$. Thus, the first interpretation with integer triangles is correct. It does not mean that the second version with the relative values is principally faulty. It only solves another task:

Instead of the initial side $d=1 ; 1040$, it starts implicitly with the $\mathbf{1 0 0} \mathbf{0 0}$-times larger integer side 110 40. Subsequently, this too large side is reduced 80-times to the final integer value $D=53$. Taking into account the implicit increase and the subsequent explicit reduction, the correct final $10000 / 80=45$-times increase, forecasted by the absolute values, is obtained.

In case of the independent factorization of the initial sides $s=0 ; 3720, d=1 ; 1040$, the final values $S=56, D=53$ in P322 are not the sides of a triangle and therefore they do not obey to relation (2). In this case, the version with the increase of initial sides by factorization is supported by unambiguous values of reciprocal pairs: Actually, due to Tab. 3 in the row $N=15$, there are generating numbers $p=9, q=5$ and the reciprocal pair $x=p / q=1 ; 48, y=q / p=0 ; 3320$. From the pair and the relation (5), the width $s=(x-y) / 2=0 ; 3720<1$; of a steep unit triangle, is determined. The width $s$ can be written in an ambiguous relative form as 3720 , but the form cannot change its unambiguous absolute value which must be respected at any factorization.

It must be strictly distinguished between the formation of the tablet P322 and its later application. The case: Friberg (1981), 4] versus Friberg (2007), 9] covers the 
whole span between the formation and the application of P322. Analysis of the errors supports the simpler, more natural Friberg [4 version, suitable for construction of P322 as a table of ordered roots and squares $s, d, d^{2},\left(s^{2}\right)$ using The extended table of reciprocals. As it will be shown later, this simpler construction of P322 would last around a month. The latter version [9] would be much more time consuming as it supposed calculation of roots $S, D$ of some apparently random squares $S^{2}, D^{2}$ which was beyond the common scribe skill. It would be a great luxury to perform calculations lasting for several month (without permanent registration of the crucial parameters $S^{2}, D^{2}$ ) just for a list of 39 numerical exercises. For this purpose, The standard table of reciprocals would be absolutely sufficient.

The majority of arguments shows that the author of P322 used parallel factorization of sides $s, d$ with the same multiples $H_{s}=H_{d}=H$ which together with the factorization terms $S, D$ represent sides of an integer triangle (rectangle).

It is undeniable that original sides $s, d, h=1$ are the sides of a unit right-angled triangle. (It is indicated by their names sag, šiliptum, uš in P322 or MS 3052.) Then proportional multiples of these sides $S, D, H$ represent sides of a similar triangle (regardless of considering them triangles or not). Although P322 gives only 2 sides $S, D$, the author of the tablet had known also the third side $H$. (By factorization, all three sides were determined simultaneously. In addition, the side $H$ should be displayed in the lost part of the tablet.)

\section{Spatial and temporal requirements for a complete tablet P322}

Dimensions of a clay tablet containing the parameters of triangles $s, d, H$, $\boldsymbol{d}^{2}, \boldsymbol{S}, \boldsymbol{D}, \boldsymbol{N}$ from Tab. 4 can be determined in a similar manner as the dimension of the tablet with parameters of Tab. 3 - from the average width $6 \mathrm{~mm}$ of a sexagesimal digit, the height $5 \mathrm{~mm}$ of a row and the height $15 \mathrm{~mm}$ of headings.

According to Tab. 4, the individual parameters are expressed by the following number of digits: $s(4), d(5), H(2), d^{2}(9), S(3), D(3), N(3)$. Adding yet 8 blank one-digit-spaces between columns and at the edges of tablet, the width of the complete tablet should be less than $37 \times 6 \mathrm{~mm}=\mathbf{2 2 2} \mathbf{m m}$. (The width of the missing and remnant part should take up $15 \times 6 \mathrm{~mm}=90 \mathrm{~mm}$ and $22 \times 6 \mathrm{~mm}=$ $132 \mathrm{~mm}$, respectively.)

A heading equivalent to 3 rows and 39 rows of triangles written on the obverse and reverse side would need a tablet less than $42 \times 5 / 2 \mathrm{~mm}=\mathbf{1 0 5} \mathbf{~ m m}$ high.

The left and right side of the tablet might be hypothetically utilized for a record of generating integers $p, q$ with Akkadian headings igibu (=numerator) 


\section{PLIMPTON 322: A UNIVERSAL CUNEIFORM TABLE}

and igu (= denominator) [15], respectively. (Numbering of rows $N$ on the obverse, reverse and flank sides would be shared.) The two-place numerator, oneplace denominator and 2 blank spaces suppose less than $5 \times 6 \mathrm{~mm}=\mathbf{3 0} \mathbf{~ m m}$ of thickness, similar to that of P322.

A complete tablet, with a horizontal writing of text, could have a shape of a clay brick less than $222 \mathrm{~mm} \times 105 \mathrm{~mm} \times 30 \mathrm{~mm}$. Initial dimensions should be larger, as the wet clay volume shrinks $7-14 \%$ by drying [16].

Similar dimensions $(223 \times 90 \times 32$ in $\mathrm{mm})$ has also a completely filled vertical tablet $M S$ 3052, as it follows from its photo ( F riberg 9]). Its reverse side is oval, just like P322, but not quite symmetrical. The oval shape is no problem for a vertical tablet with a dominating verbal text. For long horizontal rows with a numerical text, however, a planar shape of all sides is preferable. (Also on the unfinished P322, the mathematical text is on its strait not oval side.) A finished table with a full text should therefore have a shape of a thin rectangular parallelepiped.

To get at least a misty insight into the time demand of calculations, Tab. 4 was obtained from relations between individual parameters and the initial integers $p, q$, using decimal addition, subtraction, multiplication and division by a simple Microsoft calculator. The same way was also used at the final transformation of decimals to sexagesimals. The results of the calculations were later checked by Excel computations which repeatedly used the worksheet functions INT(number)\& ";" \&INT(60*MOD(number;1)) for the transformation.

Computing of 15 rows of Tab. 4 took 3 hours. The decimal operations need only a "click". All results are initially registered in the calculator memory and display, and finally simply transcribed to Word tables. On the contrary, clumsy sexagesimal operations, inscriptions and transcriptions into sets of clay tablets had to last many times longer.

An experience with 39 improper fractions $x=p(1 / q)$ of Tab. 3 has shown that their calculation by a simple calculator lasted $8 \mathrm{~min}$ while by a simple manual multiplication of two sexagesimal numbers, without any technical aid, lasted $100 \mathrm{~min}$ (about 13 times longer). Then a manual computing of 15 rows of Tab. 4 could last as minimum $13 \times 3$ hours $=39$ hours. Preparation of, maybe, 6 handy tablets for parameters $\left(s, d ; S ; D ; H ; S^{2} ; s^{2}\right)$ of the same row needed at least additional $6 \times 5 \mathrm{~min}=30 \mathrm{~min}$ plus $10 \mathrm{~min}$ for one final complete tablet for the given row $N$. Then, completion of the whole Tab. 4 with its 39 rows could last as minimum

$$
127 \mathrm{~h}(=39 \times 39 \mathrm{~h} / 15+39 \text { rows } \times(40 / 60) \mathrm{h} / \text { row }),
$$

i.e., more than 3 hours per row.

So a compilation of Plimpton 322, often being interrupted, lasted not hours but weeks. A rude guess at velocity of the compilation could be 1-2 rows of the complete table of triangles per day and per one scribe. 
A time estimate of transcription of the calculated results on the final cuneiform tablet is simpler. It is sufficient to suppose similarly, as in the case of Tab. 3: one wedge per second as a velocity of script, and 15 wedges as an average per a sexagesimal numeral. According to P322 (Fig.1.B and white part of Tab.4), individual columns of parameters (within 15 rows of the extant P322) have the following quantity of numerals: $d^{2}(90), S(30), D(32), N(30)$, in total 182 numerals and $182 \times 15=2730$ wedges. It means that a transcription of 15 rows of the extant P322 lasted around 2730 s. Supposing the headings are equal to 3 rows, then their engraving lasted $2730 \times 3 / 15=546 \mathrm{~s}$. Then writing of the extant P322 took around $3300 \mathrm{~s}=55 \mathrm{~min}$. The writing of 15 rows of the original P322, including of missing columns $s, d, H$, should be managed within 2 hours. Copying a complete table with 39 rows should last around 4 hours. (Formation of a clay tablet and its preliminary vertical and horizontal scoring could last no more than half an hour.) All copying in a hot climate should be carried out at once, within a half-day during which a clay tablet is suitable for engraving, i.e., till its surface gets "skinny" (a half way between wet and dry). In such case, an observed unified manuscript of the word $k i$ or the same depth of wedges in numbers [10, [16] can be expected.

Established time for writing the complete table (several hours) is negligible against the computational time (weeks). Then there is no problem to compile varied types of tables from all laboriously calculated data: e.g., unit triangles for builders and surveyors $(s, d, h=1, N)$; for mathematicians wanting to have a table of square roots and reciprocal pairs for solving quadratic problems $\left(x, y, s, d, d^{2}, N\right)$; for teachers wanting to have material for training of parallel factorization leading to integer triangles (triplets) $(s, d, S, D, H, N)$, suitable for computing squares of multidigital numbers $\left(d^{2}, S^{2}, 1 / H, N\right)$. The most useful is a complete set $s, d, H, \boldsymbol{d}^{2}, \boldsymbol{S}, \boldsymbol{D}, \boldsymbol{N}$. Its universal part $s, d, H$ should have been on the missing part of P322 and would have required (see above) less than $90 \mathrm{~mm}$ from the whole expected width $222 \mathrm{~mm}$ of the complete tablet. Any attempt to estimate the missing contents is speculative.

There is an argument against the complete universal table that no extant duplicate of it has been found, compared to many hundreds duplicates of other types of standard mathematical or metrological tables [7. The lack of the duplicates can be explained by a few numbers of specialized users, too little duplicates and consequently, a small probability of their preservation and excavation. Moreover, even if some duplicate or its part was found it might not have been deciphered. (Plimpton 322 itself was for decades taken to be a commercial account.) There is also a sad possibility that a propagation of the table from the ancient city Larsa could be stopped out by the capture of the city by Hammurabi's warriors. (The "circles of Archimedes" had also been forever disrupted by a soldier during the capture of Syracuse.) 


\section{PLIMPTON 322: A UNIVERSAL CUNEIFORM TABLE}

\section{Conclusions}

The mathematical text of the tablet Plimpton 322 is probable an extension of an older, less precise table compiled by means of The standard table of reciprocals to solve the problems with powers, roots and right triangles. (Data from the older table might have become part of teaching texts written on the cuneiform tablets $M S$ 3052 and $M S 3971$ from Uruk. Then it seems less probable that the tablets represent a proof of the existence of a complete tablet P322 with the extended 38 rows of triangles as it is suggested by Friberg [9, p. 447] or Britt on et al. [10, p. 561].)

\section{Supposed contribution of the article}

1. Explanation of the interest in triangles with a steep diagonal which corresponds, nowadays, to angles from $45^{\circ}$ to $90^{\circ}$ : A slope of a diagonal $d$ in a unit right triangle (or rather, in a rectangle) of a width $s$ and a length $h=1$ is restricted by the interval of widths $0 \leq s \leq 1$. The restriction simplifies calculations of a table with different inclinations. (Such table of steep triangles requires checking of 191 fractions as minimum. For moderate triangles, it is up to 484 fractions.) The restriction to steep triangles is not such limiting as it might seem, since a simple rotation can change steep triangles $(s<1)$ into moderate ones $(s>1)$, with an inclination of their diagonals from $0^{\circ}$ to $45^{\circ}$.

2. A final decision that the first column of P322 contains squares of diagonals $d^{2}=1^{2}+s^{2}$ (starting with the numeral 1) and not squares of widths $s^{2}$ of a unit triangle follows from: unambiguous values of $d^{2}$; a location of fractional numerals near the broken left edge of the tablet and from a new, high-quality photo of the text on the obverse side of P322 made by $\mathrm{Ch}$. Proust where the numeral 1 in the line 12 on the left damaged edge of P322 is still visible. (The left edge on the P322 photos in the original $\mathrm{Neugebauer}-\mathrm{Sachs}$ article [1] and Casselman [8] is illegible.)

3. Comparison of a computational complexity of construction of The extended table of reciprocal pairs $x=p / q$ and $y=1 / x$ in dependency on the restriction conditions: a maximum numerator $p<p_{\max }=2 ; 30 q$ or a maximum fraction $x<x_{\max }=2 ; 30$. In the first case, it is necessary to check 191 improper fractions $x>1$. In the latter, it is 295 .

4. Determination of a computational sequence during compilation of The extended table P322: A critical analysis of errors in the existing extant of P322 has shown that by a parallel factorization of sides of a unit triangle $s, d, h$, the integer sides of a similar triangle $S, D, H$ were calculated. From those, the squares $s^{2}=S^{2}(1 / H)(1 / H)$, $d^{2}=1+s^{2}$ were later obtained. This sequence of calculations can naturally explain all 4 arithmetical errors in P322. The independent factorization of squares of diagonal $d^{2}$ and of width $s^{2}$, promoted by F r i b e r g [9], can explain only 2 of the 4 errors and fails to clarify correctness of sides $S, D$ by incorrect values of the initial squares $s^{2}, d^{2}$. Only one independent factorization of the sides $s, d$ has occurred, probably in a time stress, at calculation of the last row $(N=15)$ of P322 by two different calculators. 
5. Explanation of a paradox of factorization: On one side, the factorization of absolute numbers leads to an enlargement of a unit triangle to that with integer sides. On the other hand, the factorization of relative values of sexagesimal numbers leads to a diminishing of the unit triangle. As the table P322 has been calculated from reciprocal pairs $x=p / q, y=q / p$ by the condition $x y=1 ; 00$, only the factorization of absolute values respect this condition.

6. The broken off part of P322 with the supposed parameters $s, d, H$ could make from the complete table $P 322$ with parameters $s, d, d^{2}$ a table of $\mathbf{7 8}$ square roots of numbers which increase with an average step 0,025 in the interval from 0 to 2 .

7. The broken off part of $P 322$ with the supposed parameters $s, d, H$ could make from the complete table P322 with parameters $s, d, H, S, D$ a table of 39 unit and 39 similar integer right triangles with a diagonal steeper than that of a square (with the inclination from $45^{\circ}$ to $90^{\circ}$, increasing with an average step $1^{\circ}$ around). However, the results are applicable also for triangles with a moderate slope of diagonal (from $0^{\circ}$ to $45^{\circ}$ ). The table is potentially interesting for builders and surveyors. It represents the earliest seeds of trigonometry where angles are not measured as an arc of a unit circle but as a width of a unit triangle.

8. Bringing to attention a possible existence of the 39th row in The extended table P322 which belongs to the simplest reciprocal pair $x=y=1 ; 00$. In this row, there is a triangle with questionable sides $s=0, d=h=1$ but also roots of the number 1 and the problematic zero ("nu" = not, nothing in Sumerian).

9. Except of the known triangle 3,4,5, the integer triangles $S, D, H$ from the complete table P322 are not suitable for measurements as they change irregularly with a continuously increasing slope of their diagonal. However, the integer triplets $S, D, H$ (obtained by a parallel factorization of sides $s, d$ of similar unit triangles) were used for calculation of squares $s^{2}=S^{2}(1 / H)(1 / H) ; d^{2}=D^{2}(1 / H)(1 / H)=1+s^{2}$. The advantage of decomposition of sides $s, d$ with multidigital values to less digital triplets by the factorization manifests when the squares $s^{2}, d^{2}$ of the multidigital sides are calculated. Conversely, in case of the unit triangle $0 ; 45,1 ; 15,1 ; 00$ with few digits, compilers of P322 did not do a tedious factorization leading to the known integer triangle $3,5,4$ but they calculated the squares $s^{2}, d^{2}$ directly by multiplication of the initial sides by itself. (It is illustrated by the relative values $S=45$, a $D=115$ in the line 11 of P322.) Such direct multiplication, instead of factorization, can be expected also in the lines 18,22, 32 and 39 with the few-digit sides of unit triangles in the extended tablet P322.

10. Time demands: for compilation of The extended table of reciprocals ( $2-8$ days per scribe, depending on the used manner of preliminary checking of calculated improper fractions $x$ ); for preparation of The extended table P322 (a month, i.e., 1-2 rows per day per scribe); for transcription of currently registered results to a final tablet of the size $222 \times 105 \times 30$ in $\mathrm{mm}$ (less than a half-day). A possibility to save lengthy and tediously calculated parameters by their brief transcription might lead, besides the completion of P322, also to a creation of several types of specialized tables. 


\section{PLIMPTON 322: A UNIVERSAL CUNEIFORM TABLE}

\section{Two lessons}

Even error may be useful:

Analysis of 4 arithmetical errors in P322 demonstrated that its author(s) used the simplest procedures and made it as a table of integer triangles (triples) $S, D, H$ and not as a table of factorization terms (cores) $S, D$ generated by an independent factorization of squares of sides $s^{2}, d^{2}$ of a unit triangle (rectangle).

Writing in clay can be more durable than in a stone:

The Old Babylonian king Hammurabi and the anonymous author of Plimpton 322 were, more or less, contemporaries. The former is known for his law code carved in stone, the latter - for his numerals inscribed in the clay tablet. While Hammurabi's realm disappeared and a majority of provisions of his code has already lost their applicability, the eight-placed sexagesimal data in Plimpton 322 has sustained its validity to the last digit, even after 4000 years. Evidently, mathematics provides more lasting knowledge than legislation.

ACKnowledgements. Author's personal experience with a clay treating was obtained due to helpfulness of Mgr. Art Milan $\mathrm{H}$ anko (Škola úžitkového výtvarníctva, Bratislava). Information on clay properties provided by him and professional potters $\mathrm{Marek}$ and $\mathrm{Henrieta} \mathrm{Hauptvogl}$ influenced and changed the author's naïve minds on fabrication of P322.

Author would like to express his gratitude also to the anonymous reviewer for his valuable advice, language corrector $\mathrm{Zuz}$ an a $\mathrm{M}$ in ar echová, technical editors Ivana Geriaková and Mária Malaščuková for preparation of the final version of this article.

\section{REFERENCES}

[1] NEUGEBAUER, O.-SACHS, A. J.: Mathematical Cuneiform Texts. With a Chapter by A. Goetze. American Oriental Series, Vol. 29, American Oriental Society and the American Schools of Oriental Research, New Haven Connecticut, 1945.

[2] BRUINS, E. M.: On Plimpton 322. Pythagorean numbers in Babylonian mathematics, Proc. Akad. Wet. Amsterdam 52 (1949), 629-632.

[3] DE SOLLA PRICE, D. J.: The Babylonian Pythagorean triangle tablet, Centaurus 10 (1964), 219-231.

[4] FRIBERG, J.: Methods and traditions of Babylonian mathematics. Plimpton 322, Pythagorean triples, and the Babylonian triangle parameter equations, Hist. Math. 8 (1981), 277-318.

[5] HØYRUP, J.: Algebra and naive geometry. An investigation of some basic aspects of Old Babylonian mathematical thought, Altorientalische Forschungen 17 (1990), 262-266.

[6] JOYCE, D. E.: Plimpton 322, Department of Math. and Comput. Sci., Clark University, 1995; http:/aleph0.clarku.edu/ djoyce/mathhist/plimpnote.htm 


\title{
RUDOLF HAJOSSY
}

[7] ROBSON, E.: Neither Sherlock Holmes nor Babylon: a reassessment of Plimpton 322. Hist. Math. 28 (2001), 1-40.

[8] CASSELMAN, W.: The Babylonian Tablet Plimpton 322, University of British Columbia, Vancouver, BC, Canada, 2003; http://www.math.ubc.ca/ cass/courses/m446-03/pl322/pl322.html

[9] FRIBERG, J.: A remarkable collection of Babylonian mathematical texts, sources and studies in the history of mathematics and physical sciences, (especially, Appendix 8 Plimpton 322, a Table of Parameters for igi-igi.bi Problems), Springer, Berlin, 2007, pp. $433-452$.

[10] BRITTON, J. P.-PROUST, CH.-SHNIDER, S.: Plimpton 322: a review and a different perspective, Arch. Hist. Exact Sci. 65 (2011), 519-566.

[11] PROUST, CH.: Trouver Toutes les Diagonales. Plimpton 322: à la Recherche des Rectangles Sexagésimaux, Une Version Mésopotamienne de la Recherche des "Triplets Pythagoriciens", Images des Mathématiques, 2015.

[12] ROBSON, E.: Words and pictures: New light on Plimpton 322, Amer. Math. Monthly 109 (2001), 105-120.

[13] ABDULAZIZ, A. A.: The Plimpton 322 tablet and the Babylonian method of generating Pythagorean triples, University of Balamand, 2010, 1-34; http://arxiv.org/abs/1004.0025v1

[14] AnAgnostakis, C.-GOLdSTEIN, B. R.: On an error in the Babylonian table of Pythagorean triples, Centaurus 18 (1974), 64-66.

[15] NEUGEBAUER, O.: Mathematische Keilschriftexte. Mathematical Cuneiform Texts, Edition with Translation and Commentary in German, Zweiter Teil/Dritter Teil, Springer-Verlag, Berlin, 1973; Glossar 30, 32, 12.

[16] HANKO, M.-HAUPTVOGL, M.-HAUPTVOGL, H.: (Personal communications.)

[17] BRUINS, E. M.: Pythagorean triads in Babylonian mathematics. The Mathematical Gazette 41 (1957), 25-28.

[18] PROUST, CH.: On the nature of the table Plimpton 322, Mathematisches Forschunginstitut Oberwolfach, Oberwolfach Report 12/2011, 664-666.

[19] KARATSUBA, A.-OFMAN, YU.: Multiplication of many-digital numbers by automatic computers. Proc. of the USSR Academy of Sci. 145 (1962), 293-294.

Received November 2, 2015

\author{
Mathematical Institute \\ Slovak Academy of Sciences \\ Štefánikova 49 \\ SK-814-73 Bratislava \\ SLOVAKIA \\ E-mail: hajossy@mat.savba.sk
}

